\title{
Aerostructural Level Set Topology Optimization for a Common Research Model Wing
}

\author{
Peter D. Dunning ${ }^{1}$ \\ National Institute of Aerospace, Hampton, VA, 23666 \\ Bret K. Stanford ${ }^{2}$ \\ NASA Langley Research Center, Hampton, VA, 23681 \\ and \\ H. Alicia Kim ${ }^{3}$ \\ University of Bath, Bath, United Kingdom, BA2 7AY
}

\begin{abstract}
The purpose of this work is to use level set topology optimization to improve the design of a representative wing box structure for the NASA common research model. The objective is to minimize the total compliance of the structure under aerodynamic and body force loading, where the aerodynamic loading is coupled to the structural deformation. A taxi bump case was also considered, where only body force loads were applied. The trim condition that aerodynamic lift must balance the total weight of the aircraft is enforced by allowing the root angle of attack to change. The level set optimization method is implemented on an unstructured three-dimensional grid, so that the method can optimize a wing box with arbitrary geometry. Fast matching and upwind schemes are developed for an unstructured grid, which make the level set method robust and efficient. The adjoint method is used to obtain the coupled shape sensitivities required to perform aerostructural optimization of the wing box structure.
\end{abstract}

\section{Nomenclature}

$=$ sensitivity factor for angle of attack

$=$ vector of Doublet Lattice Method (DLM) box areas

$=$ vector defining influence of wing deformed shape on lift

$=$ compliance of the structure

$=$ pressure coefficient vector

$=$ aerodynamic influence coefficient matrix

$=$ material property tensor

$=$ number of elements attached to a node

$=$ aerodynamic load vector

$=$ body force load vector

$=$ total load vector

$=$ acceleration due to gravity

$=$ element edge length

$=$ indices

$=$ global structural stiffness matrix

$=$ stiffness matrix of an element cut by the boundary

$=$ stiffness matrix of a finite element

$=$ iteration number

\footnotetext{
${ }^{1}$ Research Scholar, peter.d.dunning@nasa.gov, AIAA Member.

${ }^{2}$ Research Aerospace Engineer, Aeroelasticity Branch, bret.k.stanford@nasa.gov, AIAA Member.

${ }^{3}$ Senior Lecturer, Department of Mechanical Engineering, h.a.kim@bath.ac.uk, AIAA Senior Member.
} 


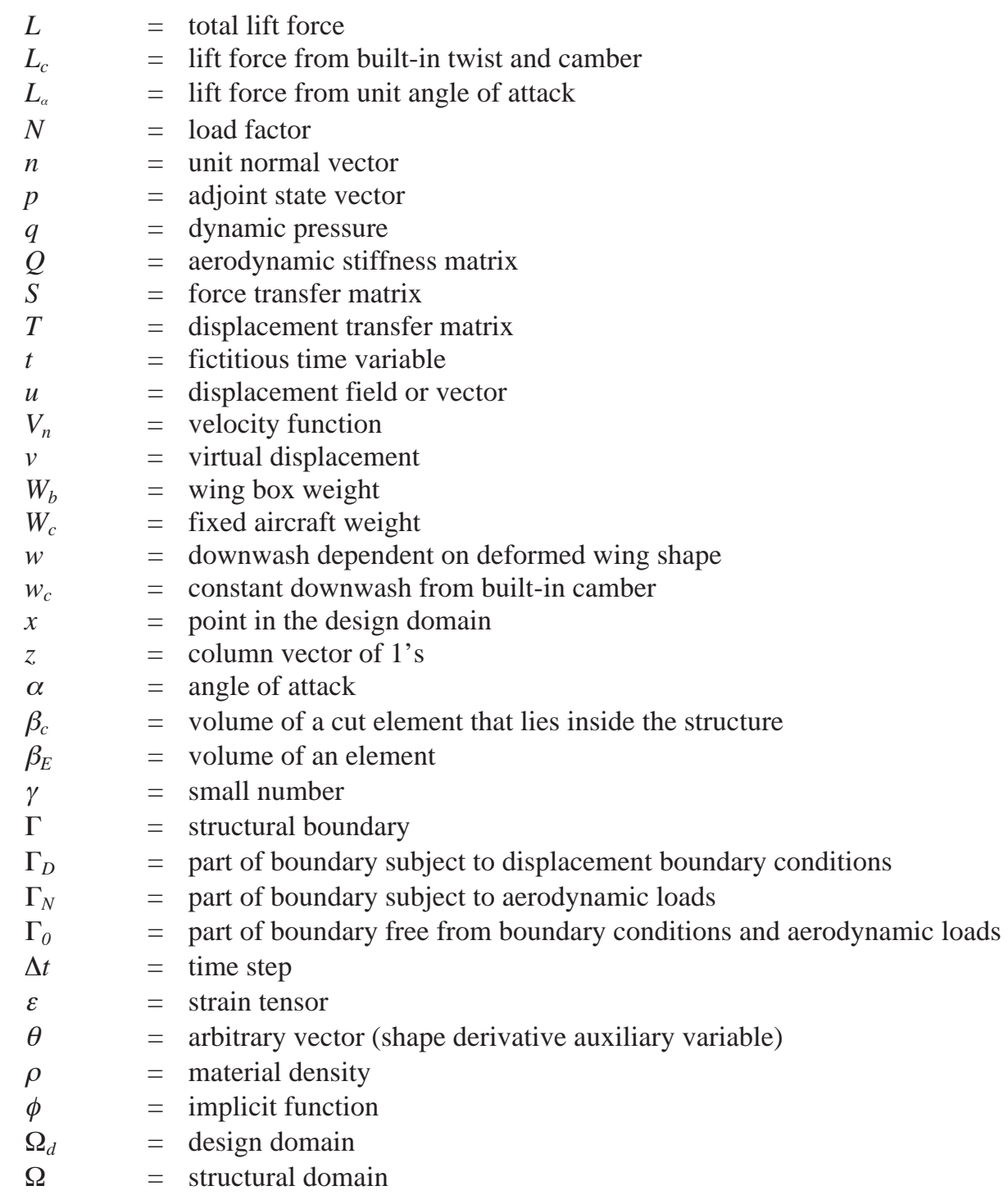

\section{Introduction}

$\mathrm{T}$

opology optimization is the most general form of structural optimization, as it is the least restricted by predetermined design variables. ${ }^{1}$ Topology optimization has been used as a tool for improving aircraft wing designs. However, the full potential of topology optimization is often not realized, as the design space is limited to a pre-determined layout or applied only to local regions or sub-components. For example, Balabanov and Haftka ${ }^{2}$ used truss topology optimization to design the internal structure of a wing, where the discrete ground structure limits the design space. The Simple Isotropic Material with Penalization (SIMP) method has been used to optimize a rib and spar type wing structure, where the layout of ribs and spars was fixed. ${ }^{3}$ The bubble method has been used to optimize the layout of a single rib for a set of loadings obtained from pull-up maneuvers and tank pressures. ${ }^{4}$ The SIMP method has also been used to optimize individual ribs. ${ }^{5}$ The spectral level set method has been applied to optimize the reinforcement of a wing upper skin to maximize aileron reversal dynamic pressure. ${ }^{6}$ Topology optimization has also been applied to the design of plate-like wings, through varying the thickness of the plate ${ }^{7,8}$ or varying the distribution of different materials. ${ }^{9}$ The motivation for this work is to consider the entire 3D wing box space as the design domain for topology optimization to explore novel configurations for the entire wing box structure.

Another important consideration in topology optimization of aircraft wings is the aerostructural coupling between the aerodynamics and the deformed shape of the wing. Early approaches computed the pressure loading for an assumed wing shape and did not account for the coupling. ${ }^{2,4}$ However, this coupling effect can strongly influence 
the optimal design. ${ }^{3}$ James ${ }^{10}$ used a multidisciplinary framework that optimized both the aerodynamic shape of the wing and the topology of the wing box structure using the SIMP method. However, the optimal designs contained a significant amount of intermediate density structure, which can be difficult to interpret as a manufacturable design, which was also noticed in Ref. 3. An alternative is to use the level set topology optimization method, ${ }^{11-13}$ which defines the boundary of the structure using an implicit function and produces solutions with clear boundaries.

Various approaches to level set based topology optimization have emerged in the last decade and there is no one method that can be called the level set method in the context of topology optimization. This review intends to provide an overview of level set based optimization. More comprehensive reviews can be found in Refs 14-16. Common to all level set methods is the representation of the boundary as the zero level set of an implicit function. Sensitivity information is then used to update the implicit function and hence the position of the boundary. The implicit boundary representation allows natural handling of topological changes, such as merging or insertion of holes, although special techniques are usually required to automatically create new holes during optimization. ${ }^{11-16}$ The initial approaches to level set based optimization used shape sensitivity analysis coupled with the level set method developed by Osher and Sethian. ${ }^{17,18}$ In this approach the implicit function is a signed distance function and it is updated by solving a Hamilton-Jacobi type equation using an explicit upwind scheme. ${ }^{11,12}$ This is sometimes called the conventional or direct approach. ${ }^{14}$ Variations on the conventional approach include solving the HamiltonJacobi equation using semi-implicit additive operator splitting ${ }^{19}$ or a semi-Lagrange approach. ${ }^{20}$ These methods can overcome the Courant-Friedrichs-Lewy (CFL) stability condition that limits the efficiency of the conventional approach. Further deviations from the conventional approach use representations of the implicit function other than the signed distance function. These include using radial basis functions, which convert the Hamilton-Jacobi PDE into an $\mathrm{ODE}^{21}$ or a simple set of algebraic equations, ${ }^{22}$ the spectral level set method, which represents the implicit function as coefficients of a Fourier series ${ }^{23}$ and methods based on phase fields, ${ }^{24,25}$ where the implicit function is used to define the material phase of a point and the update is performed by solving a reaction-diffusion equation.

The application of the level set method for wing structure optimization has been limited to local regions ${ }^{6}$ or did not account for the aerostructural coupling. ${ }^{26}$ In our previous work, the level set method was used to optimize the internal structure of a 3D wing box and included the effect of aerostructural interaction through coupled analysis and coupled sensitivities. ${ }^{27}$ However, the effect of body force loading was not included, the level set method was implemented on a uniform Cartesian grid and the examples were limited to cuboid design domains. Also, the angle of attack was fixed and the trim condition that lift must equal to weight was imposed as a constraint.

To optimize the internal structure of more realistic wings, such as the Common Research Model (CRM) ${ }^{28}$ a level set method for more general design domains that are non-rectangular needs to be developed. One approach is to immerse the general design domain in a larger uniform level set grid and maintain the shape of the actual design domain by fixing the implicit function in the region of its boundary. ${ }^{29}$ Another approach is to map the general design domain to a uniform grid, for example by using an isoparametric transform. ${ }^{26}$ However, this is only applicable when a mapping is available and the grid is structured. A third approach is to implement a level set framework that can naturally handle unstructured grids within arbitrary domain shapes, which, to our knowledge, has not been done for level set based topology optimization. In this work methods are developed to adopt the third approach.

This paper presents a strategy for optimizing the internal structure of a wing box using the conventional level set topology optimization method. The effect of the coupled aerodynamic loading is taken into account through a multidisciplinary analysis of the system and a coupled adjoint sensitivity analysis. The design space for the 3D level set optimization is the volume contained within the wing box and the optimization can choose any structure within that space. Thus, the approach is not limited by any pre-determined structural layout, such as ribs and spars. The design space is discretized using a grid fitted to the design domain, which will be non-uniform and may also be unstructured. Therefore, level set strategies for non-uniform and unstructured grids are employed.

\section{Level Set Topology Optimization}

A 3D level set method was used to optimize the internal structure of the wing box. A conventional level set method was employed. The basic method is briefly outlined followed by details of the implementation for an unstructured grid. First, the boundary of the structure is defined as the zero level set of an implicit function:

$$
\left\{\begin{array}{l}
\phi(x) \geq 0, x \in \Omega \\
\phi(x)=0, x \in \Gamma \\
\phi(x)<0, x \notin \Omega
\end{array}\right.
$$


where $\phi(x)$ is the implicit function and $x \in \Omega_{d}$, where $\Omega_{d}$ is the design domain containing the structure, $\Omega \subset \Omega_{d}$. The implicit description of the structure allows the boundary to break and merge naturally, allowing topological changes to occur during optimization.

The level set description of the structural boundary can be used for optimization. ${ }^{11-13}$ First, a velocity field is derived from shape sensitivity analysis. The implicit function is then updated by solving a Hamilton-Jacobi type equation:

$$
\frac{\partial \phi(x)}{\partial t}+\nabla \phi(x, t) \frac{d x}{d t}=0
$$

where $t$ is a fictitious time domain. Equation (2) can be discretized and rearranged to provide a convenient update formula for optimization:

$$
\phi_{i}^{k+1}=\phi_{i}^{k}-\Delta t\left|\nabla \phi_{i}^{k}\right| V_{n, i}
$$

where $i$ is a discrete point within the domain, $k$ is the current iteration number, $V_{n}$ is a velocity function defined normal to the boundary, such that a positive velocity moves the boundary inwards, and $\Delta t$ is a discrete time step. Note that Eq. (3) is the same as solving Eq. (2) using an explicit forward Euler scheme. ${ }^{18}$

\section{A. Fixed Grid Finite Element Analysis}

The design domain is discretized using isoparametric hexahedral elements with the implicit function defined at the nodes. The same grid is also utilized as the mesh for the finite element analysis (FEA) of the wing box. Elements with incompatible modes are used to analyze the wing box structure, as these elements perform better in bending compared with standard tri-linear isoparametric elements. ${ }^{30}$

The implicit function is interpolated within elements using standard tri-linear shape functions, which is useful for finding the exact position of the boundary. The implicit function is initialized as a signed distance function, where the value of the implicit function indicates the distance to the boundary and the sign is defined by Eq. (1). Details of how the signed distance function is computed on an unstructured grid are included in Section II.B.

For efficiency, the finite element mesh remains fixed during optimization. Therefore, some elements can be cut by the structure boundary leading to a discontinuity in material properties within the element. Discontinuous elements are treated using a volume-fraction weighted approximation for the element stiffness matrix:

$$
K_{c}=\left(\beta_{c} / \beta_{E}\right) K_{E}
$$

where $K_{c}$ is the approximated stiffness matrix of the cut element, $K_{E}$ is the stiffness matrix of the same element without a cut, whose volume is $\beta_{E}$, and $\beta_{c}$ is the volume of the cut element that lies inside the structure. To compute the internal volume of a cut element, $\beta_{c}$, the hexahedral element is first divided into 24 sub-tetrahedra, Fig. 1 . The implicit function is then interpolated onto the vertices of each sub-tetrahedron. The internal volume for each sub-tetrahedron can then be analytically computed and summed to obtain total value for the element. This is similar to the method proposed by Min and Gibou, ${ }^{31}$ although their method used five sub-tetrahedra. The choice of 24 subtetrahedra eliminates directional bias from the interpolation and leads to a more robust method, at additional computational expense. The method of subdividing elements into simplexes (triangles in 2D and tetrahedra in 3D) can also be used to perform surface integration when the boundary is represented by an implicit function. ${ }^{30}$

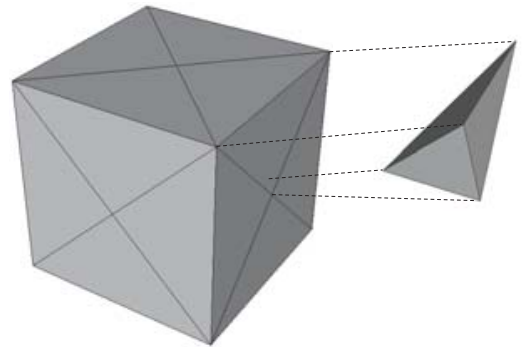

Figure 1. Dividing a hexahedral element into 24 sub-tetrahedra.

The total volume of the element, $\beta_{E}$, can be efficiently computed using an analytical formula. ${ }^{31}$ Elements that lie completely outside the boundary are assigned a small volume fraction, $10^{-6}$. This is to avoid numerical difficulties involved when part of the structure becomes completely detached from the main structure, such that it can display rigid body motions. 
It is known that using the volume fraction method of Eq. (4) (also called the ersatz material approach) leads to reduced accuracy at the boundary due to the approximation of the element stiffness. ${ }^{33-35}$ This is a concern for conventional level set methods, as the optimization is driven by shape sensitivities defined at the boundary. To overcome this problem, sensitivity values are computed at element Gauss points and interpolated at the boundary point using a weighted least squares method. ${ }^{34}$ The original method uses all Gauss point sensitivities within a specified radius of a boundary point. This is modified for a non-Cartesian mesh by using Gauss point sensitivities inside elements that neighbor the element where the boundary point is located. A 2D example is shown in Fig. 2.

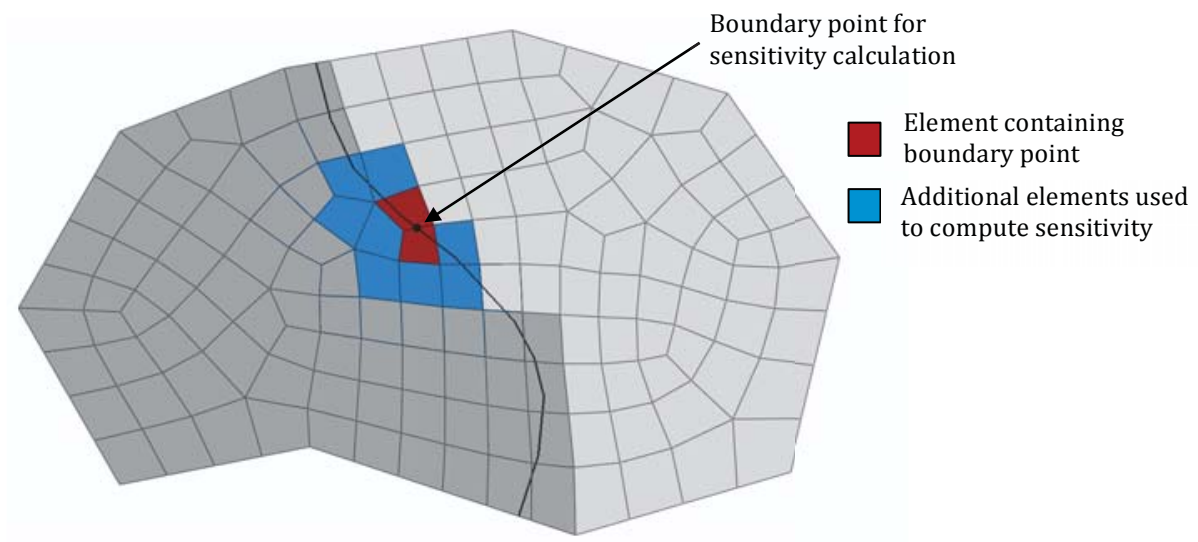

Figure 2. Boundary point sensitivity calculation for 2D unstructured mesh.

Boundary shape sensitivity values are computed at points where the boundary intersects an element edge and at grid nodes where the implicit function is zero. Boundary point shape sensitivities are then used to compute the velocity function. The derivation of the velocity function from shape sensitivities for the problem studied in this work is detailed in Section III.C.

\section{B. Velocity Extension and Reinitialization}

A key aspect of conventional level set based optimization is the definition of the velocity function from the shape derivative. However, the resulting velocity function is only defined on the structural boundary. To update the level set function using Eq. (3) discrete velocity values, $V_{n, i}$ are required at all grid points $i$. For stability, it is desirable to define these velocity values such that the signed distance property of the implicit function is maintained during the update. ${ }^{17-18}$ This can be achieved by extending the velocity to be constant along a line normal to the boundary. The velocity extension can be obtained by numerically solving: ${ }^{18}$

$$
\left(\partial V_{n} / \partial t\right)+\operatorname{sign}(\phi)(\nabla \phi /|\nabla \phi|) \cdot \nabla V_{n}=0
$$

One method to solve Eq. (5) is to choose an initial solution $V_{n}$ and then employ an iterative procedure until convergence. This is performed in a pseudo-time domain that is different from the time axis used in the main level set evolution, Eq. (2). However, this approach can be computationally expensive. On Cartesian or rectilinear grids the velocity extension can be more efficiently computed using the fast marching method. ${ }^{17}$ The fast marching method has also been generalized by Sethian and Vladimirsk ${ }^{36}$ to solve Eikonal equations on unstructured grids. Eikonal equations take the form:

$$
\|\nabla \phi\|=F(x) \text { in } \Omega
$$

where $F(x)$ is an arbitrary function. If $F(x)=1$, then the solution of the Eikonal equation can be used to reinitialize the implicit function as a signed distance function, where the magnitude of the implicit function is found from solving Eq. (6) and the sign is defined by Eq. (1). However, the unstructured grid fast marching method for solving Eikonal equations requires modification to obtain extension velocities. The method used in Ref. 36 to solve the Eikonal equation for reinitialization is first summarized followed by details of how it is modified in this work to compute the extension velocities. 
The fast marching method solves the Eikonal equation using an upwind finite difference scheme. The term upwind means that implicit function values are computed from information at points closer to the boundary. Thus, the solution is constructed in a progressive manner by marching out from the boundary. Finite differences are used to compute the spatial gradients of the implicit function. During reinitialization, the implicit function value at a point is computed by solving:

$$
\nabla \phi_{i} \cdot \nabla \phi_{i}=1
$$

where the information used to construct the gradients is obtained from local points closer to the boundary. Thus, these points should already have implicit function values computed earlier, as they are closer to, or on the boundary. In $3 \mathrm{D}$, up to three local points are used, as there are three spatial dimensions. If a grid node is near the boundary then the local points are locations where the boundary crosses an element edge attached to the node. Interpolation between two neighboring grid nodes with opposite implicit function sign is used to compute the location of a boundary point. If a grid node is not near the boundary, then the local points will be the grid nodes connected to the current node that are closer to the boundary. Due to the upwind scheme, the local points will have known implicit function values, computed during a previous step of the fast marching method.

To solve Eq. (7), a simplex is formed using the current grid

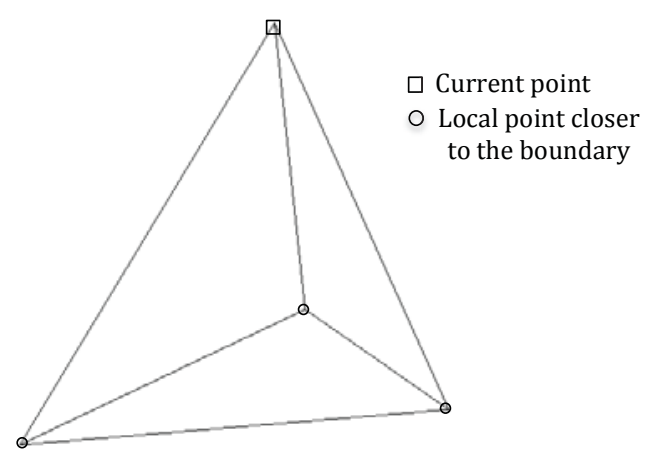

Figure 3. Local calculation of extension velocity in 3D. point and the local points closer to the boundary, Fig. 3. The spatial gradients within the simplex are assumed constant and computed using linear shape functions, which is the same as using first order finite differences. For example, if the simplex is a tetrahedron, Fig. 3, the gradients of the implicit function are computed by obtaining the isoparametric gradient matrix, $[B]$, using the four-node tetrahedron shape functions: $:^{30}$

$$
\nabla \phi_{i}=[B]\{\phi\}
$$

where $\{\phi\}$ is a vector of nodal values associated with the tetrahedron, Fig. 3. Using Eq. (8), Eq. (7) becomes:

$$
\nabla \phi_{i} \cdot \nabla \phi_{i}=\{\phi\}^{T}[B]^{T}[B]\{\phi\}=1
$$

The only unknown in Eq. (9) is the implicit function value at the current grid point, which can be obtained by solving a quadratic equation.

Equipped with a procedure for computing local solutions to Eq. (7), the fast marching method can be summarized as: ${ }^{36}$

1. Compute local solutions for grid nodes with local points on the boundary, tag these as "known."

2. Find all "unknown" grid nodes connected to the "known" set, tag these as "considered."

3. Compute local solutions for the "considered" set of nodes.

4. Find the node in "considered" with the smallest magnitude of $\phi$, tag this as "trial"

5. Find all nodes connected to "trial" that are not in "known" and update their local solutions, add these to the "considered" set and move the "trial" node to the "known" set.

6. If the "considered" set is empty, stop. Otherwise return to step 4.

The algorithm employed to compute extension velocities is developed from the fast marching method used to solve the Eikonal equation for reinitialization. The algorithm is similar and employs the same upwind and finite differencing techniques. The main difference is that instead of locally solving Eq. (7) for the implicit function value, the following is solved for the velocity function value at the current grid node:

$$
\nabla \phi_{i} \cdot \nabla V_{n, i}=0
$$


where the spatial gradient of the velocity function is computed using linear shape functions as before:

$$
\nabla V_{n, i}=[B]\left\{V_{n}\right\}
$$

where $\left\{V_{n}\right\}$ is a vector of nodal velocity values associated with the locally constructed simplex. From Eqs. $(8,10 \&$ 11) we obtain:

$$
\nabla \phi_{i} \cdot \nabla V_{n, i}=\{\phi\}^{T}[B]^{T}[B]\left\{V_{n}\right\}=0
$$

Due to the upwind scheme, the only unknown in Eq. (12) is the velocity value at the current node. Thus, Eq.(12) can be rearranged and solved for the velocity value at the current node.

Care must be taken when using the fast marching method on an unstructured grid, as the causality in the upwind scheme can be invalidated due to the presence of obtuse angles, which can cause numerical instability. ${ }^{36}$ In our implementation, this problem is partially alleviated by removing local points from the gradient approximation that cause large angles at the current point. This is a simple method and is not considered robust, as the resulting spatial gradient can be very different from the true gradient. ${ }^{36}$ However, for the grids used in this work, experience showed this method to be sufficient. More robust procedures can be found in Ref. 36.

The velocity extension method aims to preserve the signed distance function. However, the implicit function may still lose the signed distance property during optimization, due to the approximations in the numerical implementation, such as the use of first order finite differences and the heuristic method to deal with obtuse angles. Therefore, the implicit function is reinitialized as a signed distance function occasionally during the optimization.

\section{Gradient of the Implicit Function}

The spatial gradient of the implicit function at each grid node is also required to update the structure using Eq. (3). Upwind schemes are favored when solving the Hamilton-Jacobi equation, as gradients are computed according to the direction that information is traveling, which promotes stability in the solution. ${ }^{17-18}$ On a rectilinear grid, upwind schemes require a simple choice between forward or backward finite differences. However, on an unstructured grid, this is not always possible.

For an unstructured grid, spatial gradients can be computed using element shape functions in the same way that strain values are computed from nodal displacement values in FEA. ${ }^{30}$ However, gradients computed using trilinear shape functions are only guaranteed to be continuous within an element and can be discontinuous across element boundaries. Therefore, as grid nodes are attached to several elements, the gradient calculated at the same node using different elements is not certain to be the same. In FEA, a simple remedy is to average the gradient values from each element at the node. However, we would like to compute gradients according to an upwind scheme to promote stability in the level set method. Therefore, nodal gradients are computed using only the attached element from where information flows into the node. To decide which element to use, first the implicit function value at each element center is computed. Then, for node $i$, the following criterion is used to decide which element is used to compute the gradient at the node:

$$
\max _{j}\left(\operatorname{sign}\left(V_{n, i}\right) \phi_{j}\right), \quad j=1 \cdots e
$$

where $j$ is an element attached to node $i, \phi_{j}$ is the implicit function value at the center of element $j$ and $e$ is the number of attached elements. The spatial gradient of the implicit function is then computed at the node using information from the chosen element, i.e. the one that satisfies Eq. (13).

\section{The Level Set Topology Optimization Algorithm}

The time step in Eq. (3) is computed using the well-known CFL condition for stability:

$$
\Delta t=\min _{i}\left(0.9 h_{\min , i} / V_{n, i}\right)
$$

where $h_{\min , i}$ is the shortest element edge length connected to node $i$.

A simple heuristic termination criterion is used, where the optimization is terminated if the maximum relative change in the objective over the previous ten iterations is less than a small number: 


$$
\left(C_{\max }^{i}-C_{\min }^{i}\right) /\left(C_{\max }^{i}+C_{\min }^{i}\right)<\gamma, i \in[k-9, k]
$$

where $C$ is the total compliance value, or objective, $k$ is the iteration number and $\gamma$ is a small number.

Finally, the optimization algorithm is composed of the following major steps:

1. Assemble the global stiffness matrix from volume ratio weighted element matrices, Eq. (4).

2. Solve the primary and adjoint equations (Section III.D).

3. Check for convergence using Eq. (15). Otherwise continue.

4. Compute shape sensitivities at the boundary (Section II.A)

5. Velocity extension using the fast marching method (Section II.B)

6. Spatial gradient calculation using an upwind scheme (Section II.C)

7. Update the implicit function, Eq. (3)

\section{Aerostructural Optimization Problem}

The objective studied in this work is to minimize the compliance of the internal wing box structure. The loading on the structure is composed of the lift force generated by a single flight condition and the weight of the wing. The lift distribution is dependent on the deformed shape of the wing, which is in turn dependent on the applied loading. Therefore, the structure is part of a coupled, multidisciplinary system. The wing is trimmed by allowing the root angle of attack to change so that the total lift force is equal to the total weight of the aircraft. The problem can be stated as:

$$
\begin{aligned}
\text { Minimize : } & C(u)=f_{t}^{T} u \\
\text { Subject to : } & K u=f_{t}=f_{a}(u)+N \cdot f_{g} \\
& L(u)=N \cdot\left(W_{b}+W_{c}\right)
\end{aligned}
$$

where $u$ is the deformation vector of the wing, $C(u)$ is the total compliance of the wing, $K$ is the structural stiffness matrix, $f_{a}(u)$ is a load vector resulting from the aerodynamic lift force, $f_{g}$ is the body force loading due to the weight of the wing, $N$ is a load factor ( $N=1$ for steady level flight), $L(u)$ is the total lift generated by the deformed wing shape, $W_{b}$ is the weight of the wing box that can change during the optimization (and is dependent on the position of the structural boundary), whereas $W_{c}$ is the remaining "fixed" weight of the aircraft. The first constraint in Eq. (16) is the static equilibrium of the aerostructural system and the second constraint is the trim condition. The aerodynamics and pitch-based trim conditions from a tail are not considered for the wing optimization. For the taxibump load case, the aerodynamic forces are zero and the trim condition is not enforced.

\section{A. Aerodynamic Modeling}

The subsonic compressible aerodynamics are modeled using the Doublet Lattice Method (DLM) ${ }^{37-38}$ The wing planform is divided into discrete boxes, each with a control point at the $3 / 4$ chord position. The DLM is used to compute the pressure acting on each box for a given distribution of downwash at the control points:

$$
D c_{p}=w_{c}-\alpha \cdot z+w(u)
$$

where $D$ is a matrix of non-dimensional aerodynamic influence coefficients and $c_{p}$ is a vector of differential pressure coefficients for each DLM box, between the upper and lower wing surfaces. There are three sources of downwash at each control point, $w_{c}$ is the slope of the aerofoil centerline (from built-in camber and twist), $\alpha$ is the angle of attack at the wing root, $z$ is a column vector of 1's and $w(u)$ is the additional downwash dependent on the deformed shape of the wing. The total lift force generated by the wing is computed from the DLM box planform areas, the pressure coefficients and the dynamic pressure:

$$
L(u)=q \cdot a^{T} c_{p}=q \cdot a^{T} D^{-1}\left\{w_{c}-\alpha \cdot z+w(u)\right\}
$$

where $q$ is the dynamic pressure and $a$ is a column vector containing the DLM box areas. 


\section{B. Coupling the Structural and Aerodynamic Analyses}

The Finite Plate Spline (FPS) method $^{39}$ is used to communicate information between the FEA and the DLM meshes. A flat plate finite element mesh is constructed between the DLM and FEA discretizations and displacement constraints are enforced at locations that coincide with the DLM box centers and a subset of the FEA nodes. Solving the FPS finite element equation under these constraints allows the formulation of two transfer matrices that relate aerodynamic and structural quantities:

$$
\begin{aligned}
& f_{a}=q S c_{p} \\
& w(u)=T u
\end{aligned}
$$

where $S$ is a matrix that computes the work-equivalent loading on the structure from the pressure coefficients and $T$ is a matrix that converts the structural displacement vector into the downwash component dependent on the deformed wing shape, also in a work-equivalent way. Discrete Kirchoff Triangular (DKT) plate elements ${ }^{30}$ are used to form the FPS mesh.

Combining the trim condition from Eq. (16) with Eqs. (18) and (20) results in an expression for the required angle of attack for trim in terms of the deformed shape of the wing and current wing box weight:

$$
\alpha(u)=\left(L_{c}+b^{T} u-N \cdot\left(W_{b}+W_{c}\right)\right) / L_{\alpha}
$$

where $L_{c}$ and $L_{\alpha}$ are constants defined as:

$$
\begin{aligned}
& L_{c}=q \cdot a^{T} D^{-1} w_{c} \\
& L_{\alpha}=q \cdot a^{T} D^{-1} z
\end{aligned}
$$

and $b^{T}$ is a constant vector defined as:

$$
b^{T}=q \cdot a^{T} D^{-1} T
$$

The matrices $S$ and $T$ allow information to pass between the DLM and FEA and they can also be used to formulate an aerodynamic stiffness matrix:

$$
Q=q S D^{-1} T
$$

Note that the $Q$ matrix is constant for a given wing planform and flight condition, defined by velocity, air density and Mach number. However, the Mach number dependence is contained within $D$, whereas velocity and air density are used to define $q$. Thus, if the Mach number is changed, then the $D$ matrix has to be recomputed and inverted to update the $Q$ matrix. However, if dynamic pressure is changed then the update of $Q$ only involves a scalar multiplication of $Q$. The aerodynamic load vector in Eq. (16) can be computed from Eqs. (17), (19) and (25):

$$
f_{a}(u)=f_{c}-\alpha(u) \cdot f_{\alpha}+Q u
$$

where $\alpha(u)$ is defined by Eq. (21), $f_{c}$ and $f_{\alpha}$ are constant load vectors defined as:

$$
\begin{gathered}
f_{c}=q \cdot S D^{-1} w_{c} \\
f_{\alpha}=q \cdot S D^{-1} z
\end{gathered}
$$

The trim condition in Eq. (16) can now be eliminated and the problem re-stated as: 


$$
\begin{aligned}
& \text { Minimize : } C(u)=f_{t}^{T} u \\
& \text { Subject to: } K u=f_{t}=f_{a}(u)+N \cdot f_{g}
\end{aligned}
$$

where the aerodynamic load vector $f_{a}(u)$ is defined by Eq. (26) and the required angle of attack for trim is defined by Eq. (21). The only constraint in Eq. (29) is the static equilibrium equation for the coupled aerostructural system, which is implicitly satisfied when solving for $u$. Thus, the optimization problem defined in Eq. (29) can be solved using unconstrained methods, although it may be necessary to introduce an upper limit on the structural volume to obtain useful results. For compliance minimization problems without design dependent loading a volume constraint is necessary to prevent the trivial solution where the design domain is completely filled with material. A volume constraint is not required here, as adding weight to the wing can increase the compliance because of the design dependent loads. Adding weight will increase the aerodynamic loads, through an increase angle of attack for trim, and also increase the body force loads. An upper limit on $\alpha$ may be required to prevent wing stall, which is highly undesirable in flight. The DLM cannot model stalled flow, and this effect is not included in this work.

\section{Shape Sensitivity Analysis}

The shape derivative for the problem defined in Eq. (29) is derived using results from Ref. 10 and accounting for the influence of the aerostructural coupling and the trim condition. The compliance can be written in a continuous form as:

$$
C(\Omega, u)=N \int_{\Omega}\left(f_{g} \cdot u\right) d \Omega+\int_{\Gamma_{N}}\left(f_{c} \cdot u\right) d \Gamma_{N}-\alpha(u) \int_{\Gamma_{N}}\left(f_{\alpha} \cdot u\right) d \Gamma_{N}+\int_{\Gamma_{N}}\left(Q_{i, j} u_{i} \cdot u_{j}\right) d \Gamma_{N}
$$

where $\Gamma_{N}$ is the part of the boundary where the aerodynamic loads are applied (such as the upper surface of the wing) and $Q_{i, j}$ is the continuous tensor form of the aerodynamic stiffness matrix. The continuous form of the aerostructural equilibrium equation is:

$$
\begin{aligned}
\int_{\Omega}(E \varepsilon(u) \cdot \varepsilon(v)) d \Omega= & N \int_{\Omega}\left(f_{g} \cdot v\right) d \Omega+\int_{\Gamma_{N}}\left(f_{c} \cdot v\right) d \Gamma_{N}-\alpha(u) \int_{\Gamma_{N}}\left(f_{\alpha} \cdot v\right) d \Gamma_{N}+\int_{\Gamma_{N}}\left(Q_{i, j} v_{i} \cdot u_{j}\right) d \Gamma_{N} \\
& +\int_{\Gamma_{D}}(v \cdot E \varepsilon(u) n+u \cdot E \varepsilon(v) n) d \Gamma_{D}
\end{aligned}
$$

where $E$ is the material property tensor, $\varepsilon(u)$ is the strain tensor, $v$ is a virtual displacement, $n$ is a unit vector normal to the boundary and $\Gamma_{D}$ is the part of the boundary where displacement boundary conditions are applied.

The adjoint method is used to obtain the shape derivative of Eq. (30). First, a Lagrangian function is formed by adding the aerostructural equilibrium equation, Eq. (31), to the objective, Eq. (30), using a Lagrange multiplier, $\lambda$ :

$$
\begin{aligned}
\Lambda(\Omega, v, \lambda)= & C(\Omega, v)-\int_{\Omega}(E \varepsilon(v) \cdot \varepsilon(\lambda)) d \Omega+N \int_{\Omega}\left(\lambda \cdot f_{g}\right) d \Omega+\int_{\Gamma_{N}}\left(\lambda \cdot f_{c}\right) d \Gamma_{N} \\
& -\alpha(v) \int_{\Gamma_{N}}\left(\lambda \cdot f_{\alpha}\right) d \Gamma_{N}+\int_{\Gamma_{N}}\left(Q_{i, j} \lambda_{i} \cdot v_{j}\right) d \Gamma_{N}+\int_{\Gamma_{D}}(\lambda \cdot E \varepsilon(v) n+v \cdot E \varepsilon(\lambda) n) d \Gamma_{D}
\end{aligned}
$$

The adjoint equation is derived by differentiating Eq. (32) with respect to $v$ in the direction $\theta$ and defining $(u, p)$ as a stationary point, where $\theta$ is an arbitrary vector. After collecting similar terms:

$$
\begin{aligned}
\left\langle\frac{\partial \Lambda}{\partial v}(\Omega, u, p), \theta\right\rangle & =0=N \int_{\Omega}\left(f_{g} \cdot \theta\right) d \Omega+\int_{\Gamma_{N}}\left(f_{a}(u) \cdot \theta\right) d \Gamma_{N}-\frac{\partial \alpha(u)}{\partial u} \int_{\Gamma_{N}}\left(f_{\alpha} \cdot(u+p)\right) \theta d \Gamma_{N} \\
+ & \int_{\Gamma_{N}}\left(Q_{i, j}\left(u_{i}+p_{i}\right) \cdot \theta_{j}\right) d \Gamma_{N}-\int_{\Omega}(E \varepsilon(\theta) \cdot \varepsilon(p)) d \Omega+\int_{\Gamma_{D}}(p \cdot E \varepsilon(\theta) n+\theta \cdot E \varepsilon(p) n) d \Gamma_{D}
\end{aligned}
$$

where the chevron brackets denote a vector inner product. To complete the derivative in Eq. (33) the change in angle of attack due to a change in deformed wing shape is required. From Eq. (21):

$$
\partial \alpha(u) / \partial u=b / L_{\alpha}
$$


Using FEA to solve the adjoint equation, Eq. (33) can be written in the following matrix form:

$$
K^{T} p=N \cdot f_{g}+f_{a}(u)+\left[Q^{T}-\left(b \otimes f_{\alpha}\right) / L_{\alpha}\right](u+p), u=p=0 \text { on } \Gamma_{D}
$$

The shape derivative can now be computed by differentiating the Lagrangian, Eq. (32) with respect to the shape in the direction $\theta$, at the stationary point $(u, p)$. During optimization, the part of the boundary where boundary conditions and aerodynamic loads are applied is fixed, thus $\theta=0$ on these parts and we only compute the shape derivative on the remaining free part of the boundary, $\Gamma_{0}$ :

$$
\frac{\partial \Lambda}{\partial \Omega}(\Omega, u, p)(\theta)=\int_{\Gamma_{0}} \theta \cdot n\left(N \cdot f_{a} \cdot(u+p)-E \varepsilon(u) \cdot \varepsilon(p)\right) d \Gamma_{0}-\frac{\partial \alpha(u)}{\partial \Omega} \int_{\Gamma_{N}}\left(f_{\alpha} \cdot(u+p)\right) d \Gamma_{N}
$$

The second part of Eq. (36) is the change in the compliance with respect to a change in the angle of attack due to a change in the total weight of the structure. Thus, from Eq. (21) the derivative of $\alpha$ with respect to the shape, at the stationary point, is simply the shape derivative of the structural weight multiplied by the load factor over $L_{\alpha}$ :

$$
\partial \alpha(u) / \partial \Omega=-N / L_{\alpha} \int_{\Gamma_{0}} \theta \cdot n(\rho g) d \Gamma_{0}
$$

where $\rho$ is the density of the material and $g$ is acceleration due to gravity.

Setting $\theta \cdot n$ equal to the normal velocity, where a positive velocity is an inward movement: $\theta \cdot n=-V_{n}$, the final shape derivative on the free boundary becomes:

$$
\frac{\partial C}{\partial \Omega}(\Omega, u, p)=\int_{\Gamma_{0}} V_{n}\left(E \varepsilon(u) \cdot \varepsilon(p)-N \cdot f_{a} \cdot(u+p)-A \cdot \rho g\right) d \Gamma_{0}, \quad A=\frac{N}{L_{\alpha}} \int_{\Gamma_{N}}\left(f_{\alpha} \cdot(u+p)\right) d \Gamma_{N}
$$

where $A$ is a factor, constant over the entire boundary, that relates the change in compliance due to a change in the angle of attack, resulting from a change in the wing box weight.

To solve the aerostructural optimization problem stated in Eq. (29) using the level set method, the shape derivative must be negative to ensure a decrease in the objective. Thus, the velocity function is defined such that the shape derivative, Eq.(38) is negative everywhere on the free boundary. The velocity function is simply defined as:

$$
V_{n}=N \cdot f_{g} \cdot(u+p)+A \cdot \rho g-E \varepsilon(u) \varepsilon(p)
$$

This velocity function is used with the level set method described in Section II to progressively improve the structure with respect to the optimization objective. The sensitivity calculation was validated against sensitivities obtained using finite differences.

\section{Solution of the Primary and Adjoint Aerostructural Problems}

The aerostructural equilibrium equation, Eq.(29), can be restated as a linear equation and solved directly. However, in practice this is not an efficient approach, as the aerodynamic stiffness matrix is dense, whereas the structural stiffness matrix is sparse. Thus, methods usually employed to efficiently solve static linear elastic equations may not be efficient for solving the coupled equation, due to the dense matrix. Therefore, it can be more efficient to use an iterative approach to solve the aerostructural equilibrium equation.

The iterative approach employed in this work starts with a load vector computed assuming no coupling between the aerodynamic loads and deformed wing shape. The deformed wing shape is obtained for the current load vector, which is then used to update the load vector by including the coupling terms. This process is repeated until the difference between the previous and updated load vectors is very small. The process can be summarized as:

1. Initialize load vector: $f^{0}=N \cdot f_{g}+f_{a}(0)$

2. Solve: $K u^{k+1}=f^{k}$

3. Update load vector: $f^{\mathrm{k}+1}=N \cdot f_{g}+f_{a}\left(u^{k+1}\right)$

4. Check for convergence: $\left\|f^{\mathrm{k}+1}-f^{\mathrm{k}}\right\|<$ small 
The iterative approach is efficient, although it can be unstable and fail to converge, especially for more flexible wings. To overcome this, a not convergent solution is detected by tracking the relative difference in subsequent load vectors. If the solution is not converging, an under-relaxation approach is used to obtain the next load vector: $f^{\mathrm{k}+1}=$ $N \cdot f_{g}+0.25 f_{a}\left(u^{k}\right)+0.75 f_{a}\left(u^{k-1}\right)$. The relaxation factor of 0.25 was chosen to give a good compromise between efficiency and stability for the examples studied in this work.

The iterative approach is also used to solve the adjoint equation, Eq.(35). In this case the displacement vector, $u$, is known from the solution of the primary equation. The initial adjoint load vector is computed using: $p=0$. The adjoint solution is then obtained using the same iterative approach that is used to find the primary solution, including the under-relaxation method for stabilization.

\section{Optimization of a CRM Wing Box}

The 3D unstructured grid level set method, detailed in Section II, is used solve the aerostructural optimization problem, detailed in Section III. The subject of the optimization is the internal configuration of the CRM wing box. ${ }^{28}$

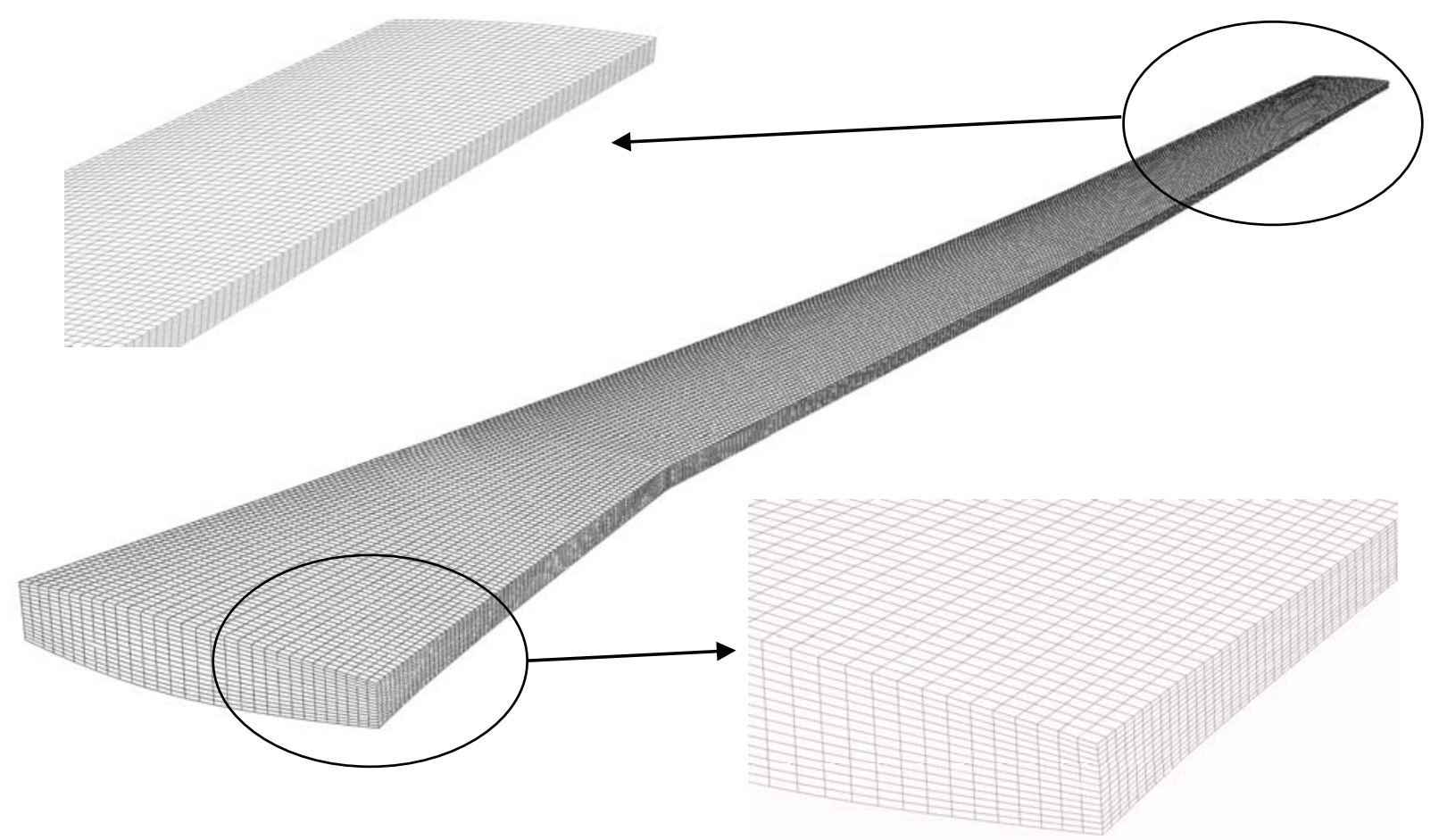

Figure 4. CRM solid wing box mesh.

\section{A. The Common Research Model Wing}

The CRM is a transonic transport configuration with an aspect ratio of 9 , a taper ratio of 0.275 , a sweep angle of $35^{\circ}$, and a cruise Mach number of 0.85 . For all flight loads a dynamic pressure of 5,897 $\mathrm{Pa}$ is used. The wing box is located between $12 \%$ and $71 \%$ of the local airfoil chord. The fixed weight of the aircraft is $500 \mathrm{kN}$, thus, in steady level flight, each wing must produce enough lift to support $250 \mathrm{kN}$ plus the weight of one wing. The wing box structure is made from aluminum, with a Young's modulus of $68.95 \mathrm{GPa}$, Poisson's ratio of 0.3 and density of 2800 $\mathrm{kg} / \mathrm{m}^{3}$.

The CRM wing box mesh is shown in Fig. 4 and has 150,300 hexahedral elements and 166,160 nodes. There are 15 elements though the depth, 30 along the chord and 334 along the span. This mesh is used for the FEA and to discretize the implicit function. All degrees of freedom for nodes at the wing root are fixed. The DLM mesh is composed of $20 \times 100$ boxes in the chord-wise and span-wise directions, respectively, Fig. 5. The FPS method is used to apply the aerodynamic loads to a subset of 1705 nodes on the upper surface of the structural mesh, Fig. 6 . When the FPS method is used to transform displacements into downwash, information is used from both the upper 
and lower surfaces of the wing box mesh. The complete aeroelastic model, described in Section III, was validated against results obtained from NASTRAN. FEA and DLM results were validated independently, before validating the whole model, including the FPS method.

The hexahedra finite elements used to discretize the wing box cannot model thin, shell-like structures typical of the conventional semi-monocoque configuration. Therefore, the structures obtained using the mesh in Fig. 4 are likely to be much heavier than real wing boxes. This will result in a high angle of attack and large body force loads, which are undesirable and make comparison to conventional configurations difficult. To obtain reasonable topologies with the mesh in Fig. 4, the modulus and density of the material used during the entire optimization are $10 \%$ of the real values.
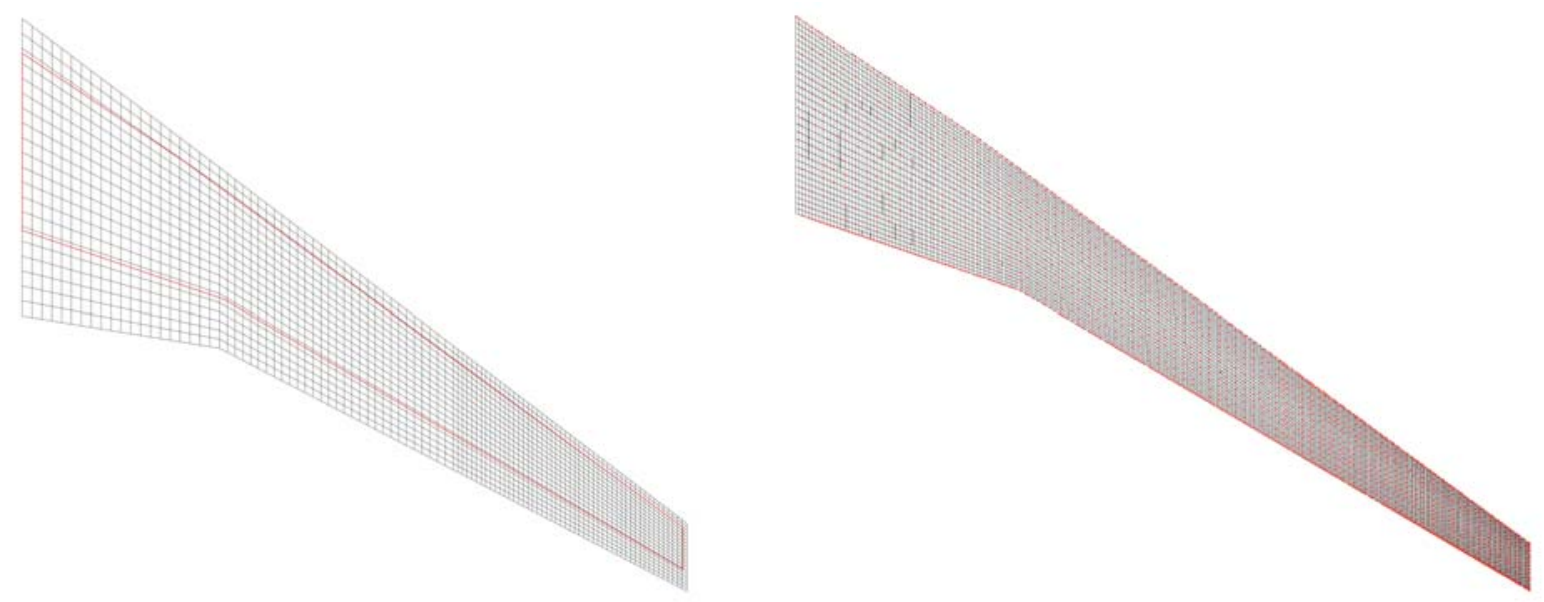

Figure 5. DLM mesh (CRM wing box outlined in red). Figure 6. Down selected nodes used in the FPS method.

The 3D level set optimization method is free to choose any structure within the wing box domain and is not restricted by any predefined configuration. However, during the optimization the outer layer of elements is fixed to remain part of the structure, therefore the upper and lower layers of elements act as the upper and lower skins. This ensures that holes do not develop in the upper and lower skins and that the skins remain connected. Finally the convergence criterion used for all examples is $\gamma=8 \times 10^{-4}$, Eq. (15). This value was chosen based on the observed convergent behavior of the examples studied in this work.

\section{B. Single Load Case}

This section considers several single load case problems for a range of flight maneuvers, characterized by the load factor, $N$, and a ground load case, which is a $2.5 \mathrm{~g}$ taxi-bump. The ground load case only considers static body force loads and does not consider any aerodynamic loading or the trim condition. The initial configuration in each case is shown in Fig. 7, where material fills the wing box with regularly distributed spherical voids. The initial voids are required, as the current method cannot automatically create new holes during optimization.

\section{Steady level flight}

The first single load case problem is steady level flight with a load factor: $N=$ 1. The solution is shown in Fig. 8. The topology of the solution is relatively simple, as material is added to the outboard region and removed from the inboard region, although there are some subtle variations of the skin thickness in the inboard area. The extra material towards the tip helps reduce compliance by inertia relief, as the increase in the body force loading outboard acts to oppose the wing bending caused by the aerodynamic lift force. Although optimal for flight, the concentration of material at

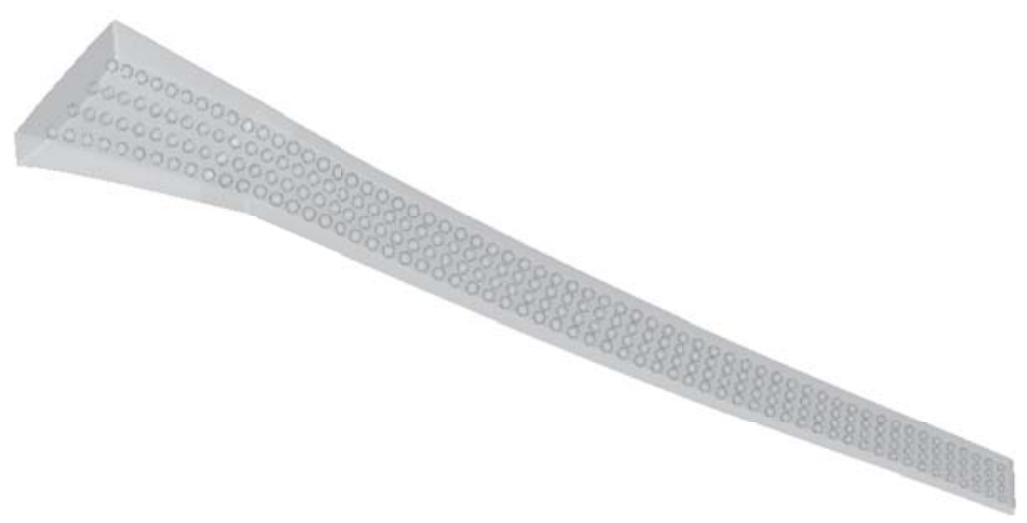

Figure 7. CRM wing box initial design. 
the tip is undesirable when considering ground load cases, such as the taxi-bump case, as the moment due to the tip mass can exceed that caused by the flight loads. ${ }^{40}$ The material removed from the inboard region also helps reduce compliance, as the wing box weight is reduced, which results in a reduced angle of attack and reduced aerodynamic loading, Fig. 9. The two primary factors driving the topology in this case appear to be an increase of body force loading outboard and an overall weight reduction to reduce the root angle of attack and associated loading. The results show no significant aeroelastic tailoring during the optimization, as the ratio of tip deflection to tip twist changes by only $2.8 \%$ from the initial to the final structure.

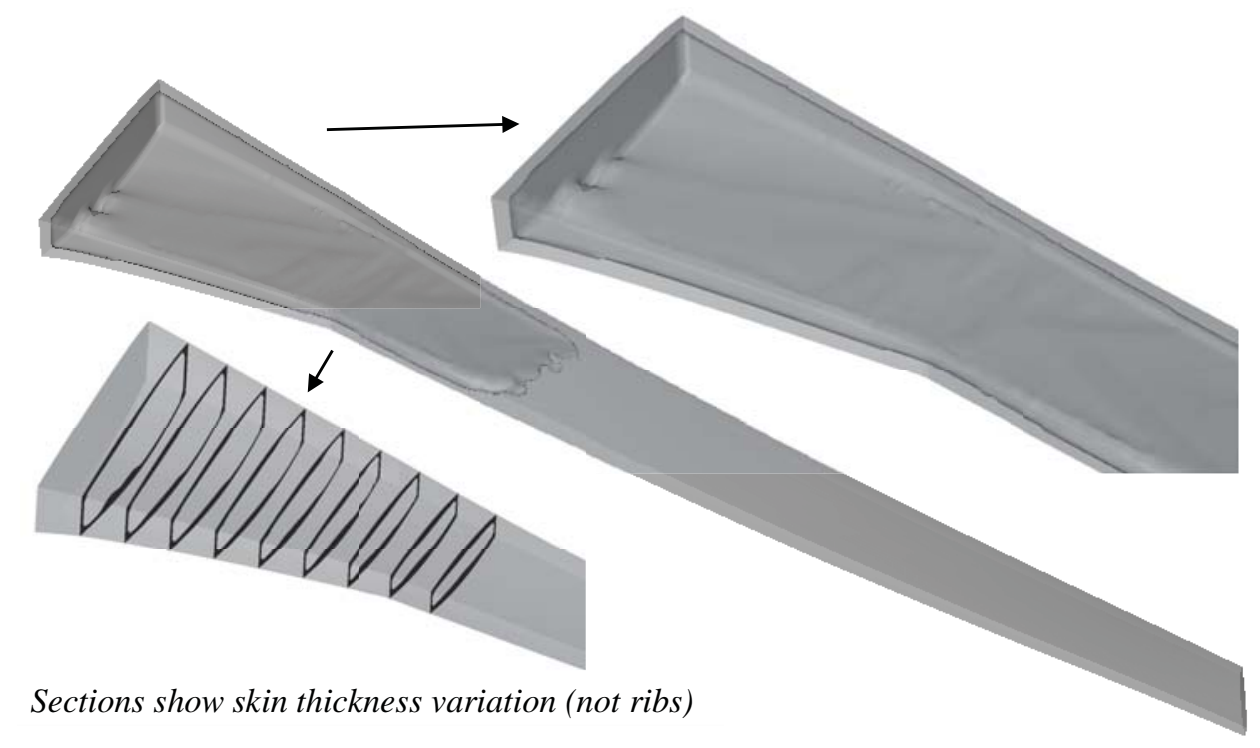

Figure 8. Steady level flight solution.

The unstructured 3D level set method performed well and the solution converged in 48 iterations, Fig. 10.

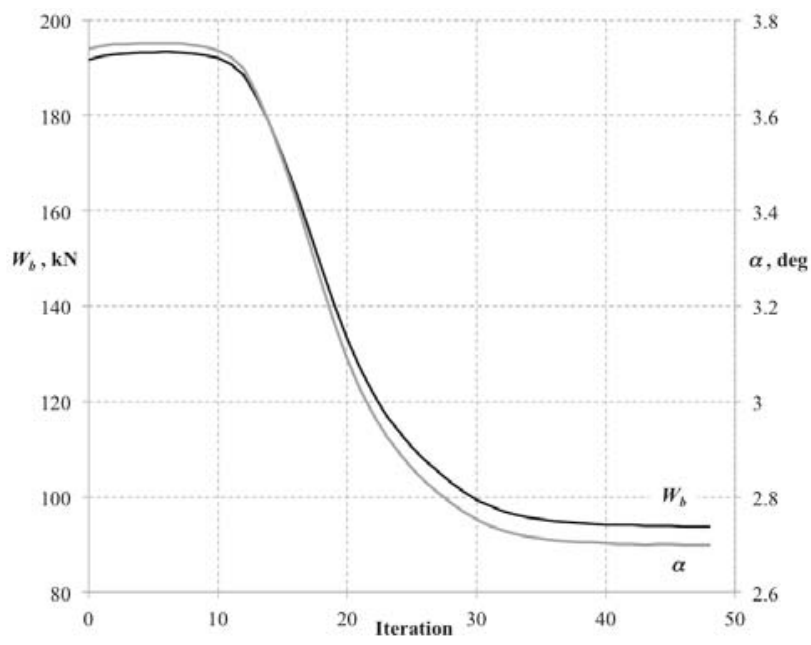

Figure 9. Steady level flight, weight and angle of attack.

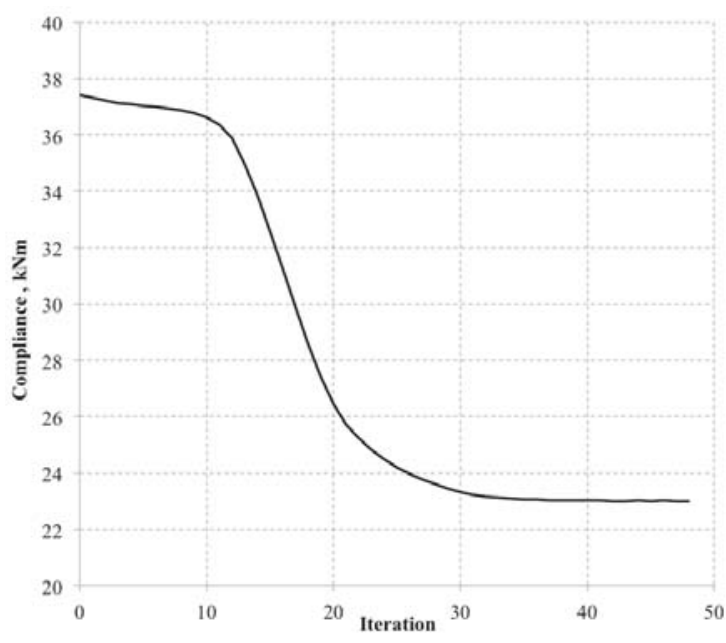

Figure 10. Steady level flight, compliance.

\section{Flight maneuvers}

Two flight maneuvers are considered, a pull-up maneuver, with $N=2.5$, and a push-over maneuver, with $N=-1$. These were chosen as they are typically used to size an aircraft at the boundaries of the flight envelope ${ }^{41}$ All other parameters are the same as for the steady level flight example. The solutions for the pull-up and push-over maneuvers are shown in Fig. 11 and 12, respectively. The topologies for the maneuver cases are very similar to the solution for the steady level flight case, Fig. 8, with only small differences in the thickness of the skins. This is not surprising, as the two main factors that drive the topology are the body force loading and aerodynamic loading from 
the root angle of attack, both of which scale linearly with the load factor. The results are not exactly the same, as the constant part of the aerodynamic load vector does not vary with the load factor. It can be seen from the convergence histories of wing box weight, angle of attack and compliance, that all flight load cases follow a similar path during optimization, Figs 9, 10, 13-16, with only a noticeable difference in the magnitudes (and sign for the push-over maneuver angle of attack).

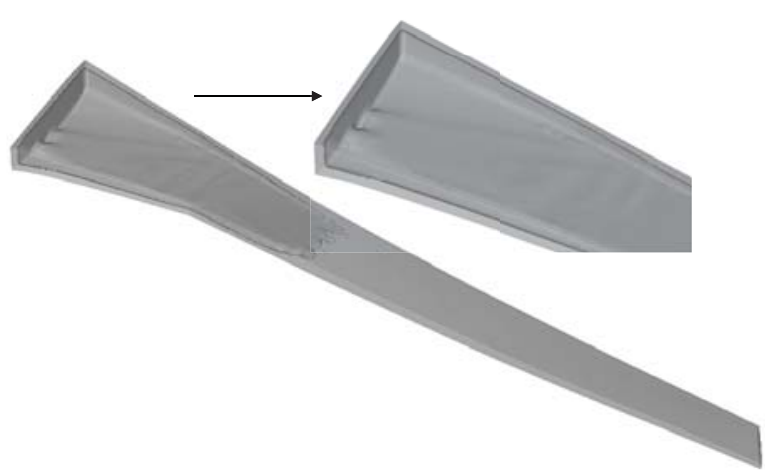

Figure 11. Pull-up maneuver solution.

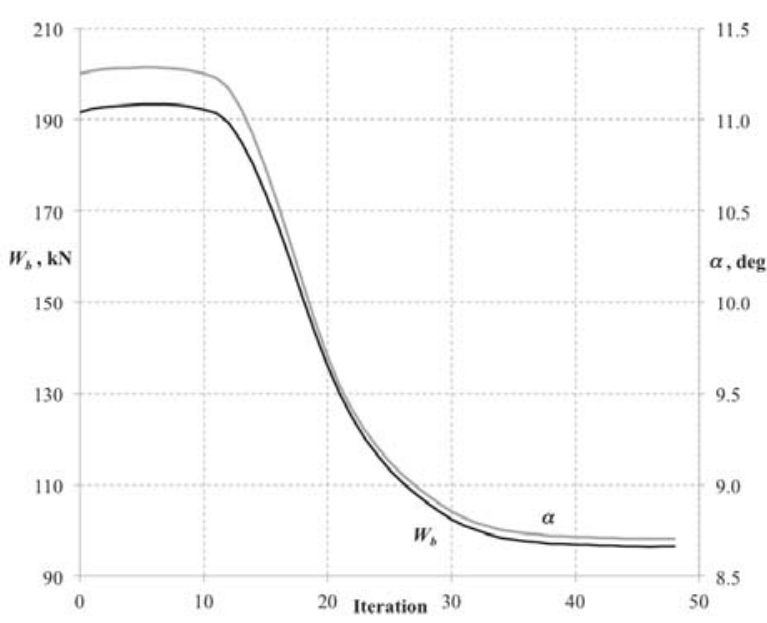

Figure 13. Pull-up, weight and angle of attack.

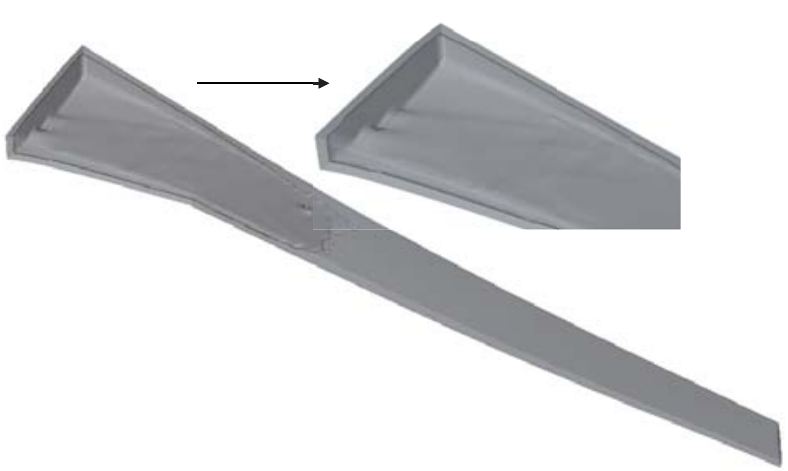

Figure 12. Push-over maneuver solution.

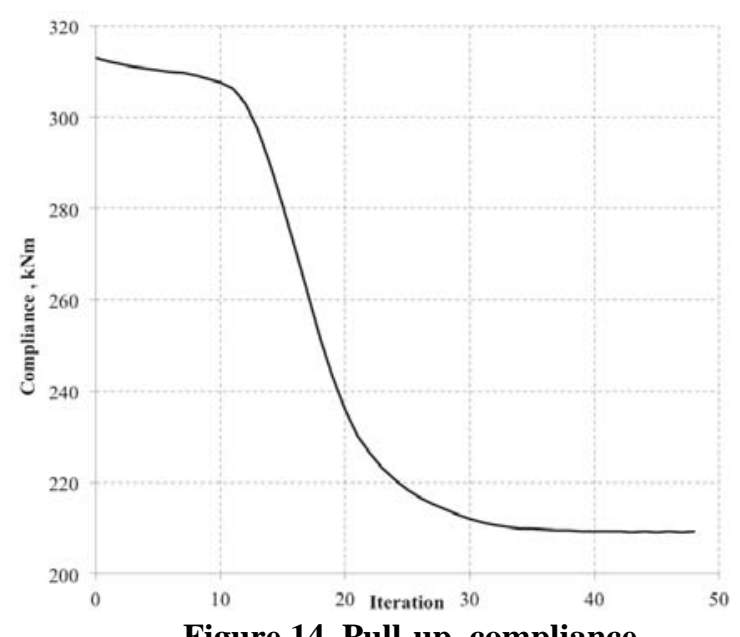

Figure 14. Pull-up, compliance.

\section{Taxi-bump}

The load factor for the taxi-bump case is $N=-2.5$. No aerodynamic loads are applied and the trim condition is not enforced. The taxi-bump case is self-adjoint, as the adjoint equation, Eq. (35), reduces to the static equilibrium equation, thus $u=p$. The solution for the taxi-bump case is shown in Fig. 17 and is very different from the flight load case solutions. Material has been removed from the outboard region to reduce the inertia effect on the bending and some material has been added to the inboard region, to reinforce the wing box against bending. Some initial voids in the inboard region persist, which suggests that the removal of these voids has little impact on the compliance. Another difference from the flight load solutions is the lack of local variations in the wing skin thickness, which suggests that this feature is a result of the aeroelastic coupling for the flight load examples. Note that the solution shown in Fig. 17 has not reached the specified convergence criterion and was terminated after 400 iterations, although the total compliance is not changing significantly at this point, Fig. 18. 


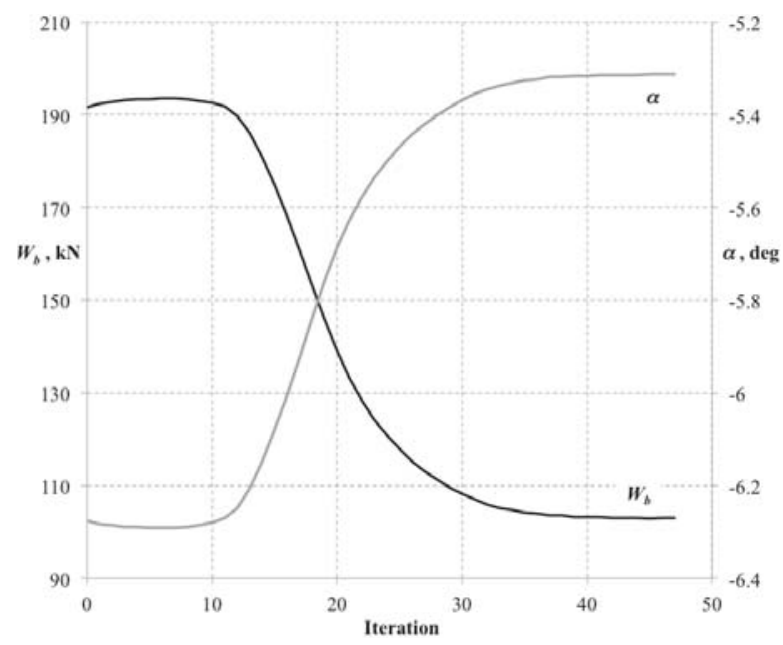

Figure 15. Push-over, weight and angle of attack.

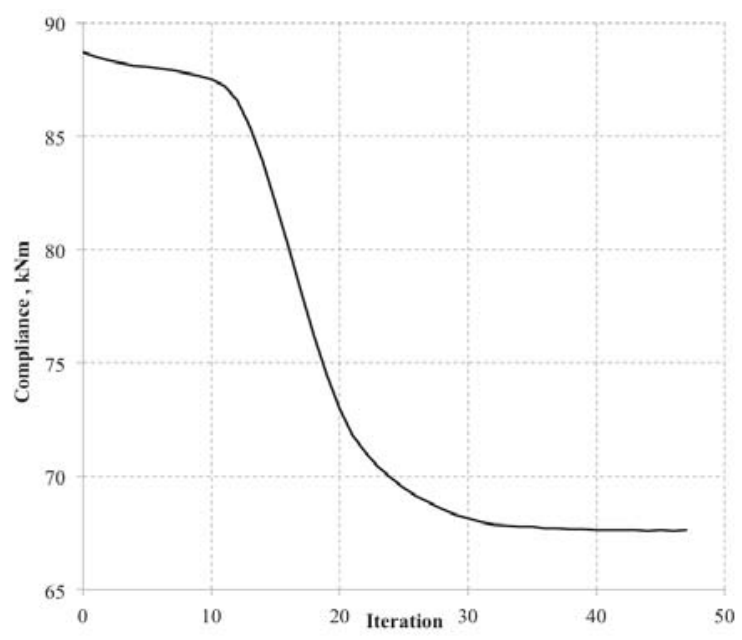

Figure 16. Push-over, compliance.

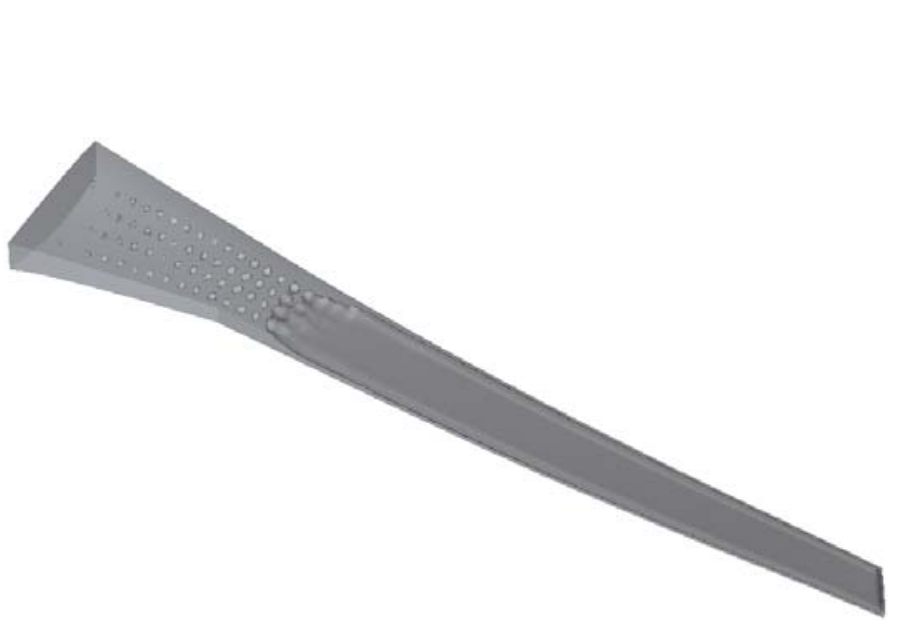

Figure 17. Taxi-bump solution.

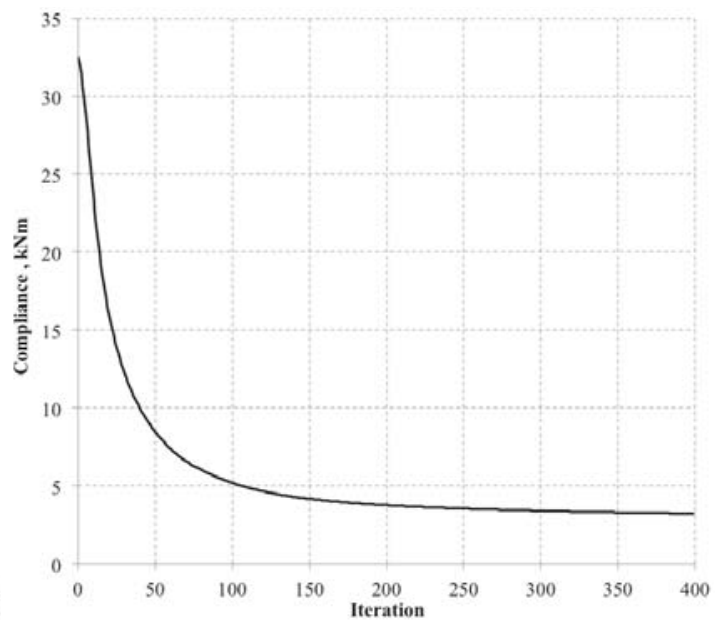

Figure 18. Taxi-bump, compliance.

\section{Multiple Load Cases}

The results in the previous section show that optimal designs for flight load cases have a concentration of material in the outboard region for inertia relief, Figs. 8, 11 and 12. This feature is undesirable for ground load cases and the optimal design for the taxi-bump case has minimal material outboard, Fig. 17. Therefore, the optimal designs for flight load and ground load cases are conflicting. The trade-off between these two load cases is explored by solving a multiple load case problem where one load case is steady level flight and the second load case is the taxi-bump:

$$
\text { Minimize }: \eta C_{f}+(1-\eta) C_{g}
$$

where $C_{f}$ is the compliance from the steady level flight case, $C_{g}$ is the compliance from the ground load taxi-bump case and $\eta$ is a weighting factor for the two cases, where $\eta \in[0,1]$. The shape derivative for the multiple load case problem is simply the weighted sum of the derivatives from each case.

The multiple load case problem, Eq. (40), was solved for five values of $\eta: 0.0,0.25,0.5,0.75,1.0$. The solution for $\eta=1.0$ is the same as the steady level flight solution, Fig. 8 , and the solution for $\eta=0.0$ is the same as the taxibump solution, Fig. 17. Solutions for the other $\eta$ values are shown in Fig. 19. Table 1 shows a summary of wing box weight values and compliance values, for each solution and both load cases, as expected the compliance for the 
flight load case increases with decreasing $\eta$ and the opposite occurs for the ground load compliance. However, the trend for wing box weight is not so clear.

Table 1. Multiple load cases, compliance and wing box weight values.

\begin{tabular}{|c|c|c|c|}
\hline $\boldsymbol{\eta}$ & $\boldsymbol{C}_{f}, \mathbf{k N m}$ & $\boldsymbol{C}_{\boldsymbol{g}}, \mathbf{k N m}$ & $\boldsymbol{W}_{\boldsymbol{b}}, \mathbf{k N}$ \\
\hline 1.00 & 23.0 & 39.8 & 93.8 \\
\hline 0.75 & 27.6 & 9.0 & 77.5 \\
\hline 0.50 & 44.0 & 5.2 & 161.8 \\
\hline 0.25 & 46.4 & 3.5 & 153.6 \\
\hline 0.00 & 50.4 & 3.2 & 151.9 \\
\hline
\end{tabular}

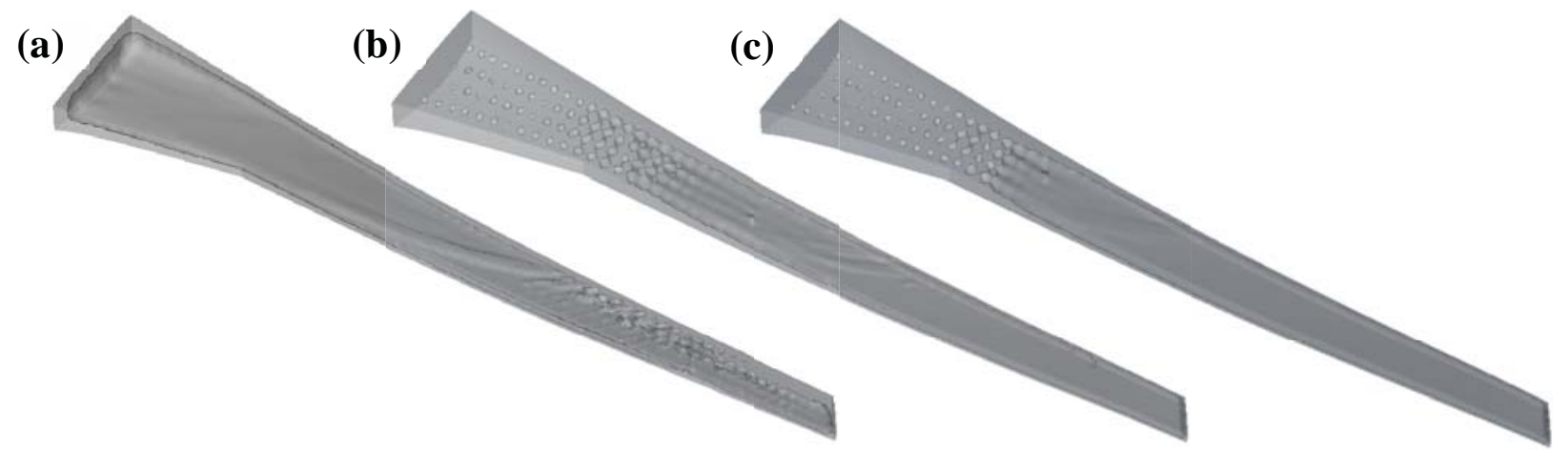

Figure 19. Multiple load case solutions: a) $\eta=0.75$, b) $\eta=0.5$, c) $\eta=0.25$.

The solutions for $\eta=0.5$ and 0.25 are similar to the taxi-bump solution, as material is removed from the outboard region. More material is removed as $\eta$ is decreased, as the inertial effects of the taxi-bump load case influence the optimization more. There are also some subtle skin thickness variations that are not seen for the pure taxi-bump solution, which may be a result of the aeroelastic coupling in the optimization. The compliance values for the $\eta=0.5$ and 0.25 solutions are within $10 \%$ of those for the taxi-bump case, where the flight load compliance is an order of magnitude greater than the ground load compliance, Table 1 . The compliance convergence history for the $\eta$ $=0.5$ problem shows that the flight load compliance actually increases during optimization, Fig. 20. The results suggests that the $\eta=0.5$ and 0.25 weighting values do not provide a good compromise between the flight and ground load cases for compliance minimization, as the solutions are not significantly different from the taxi-bump case and compliance values for the two load cases are an order of magnitude different.

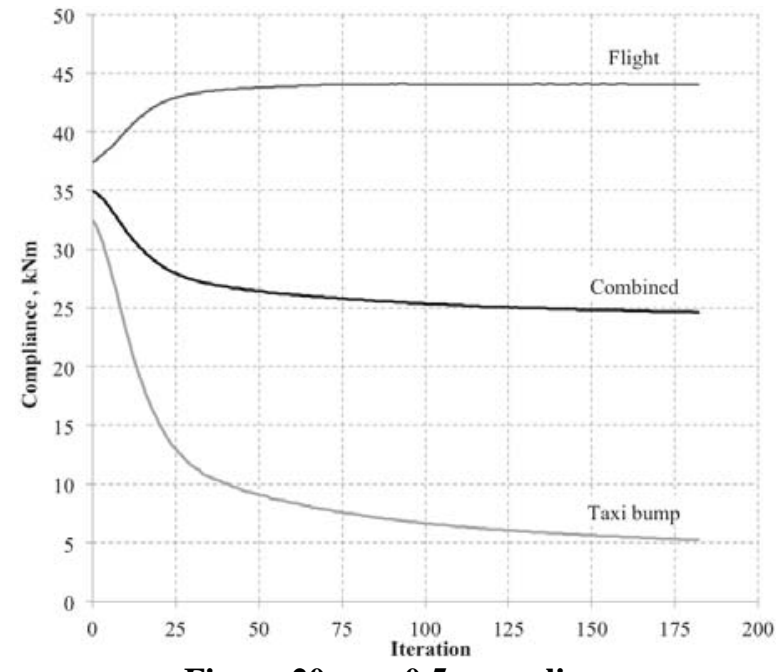

Figure 20. $\eta=0.5$, compliance.

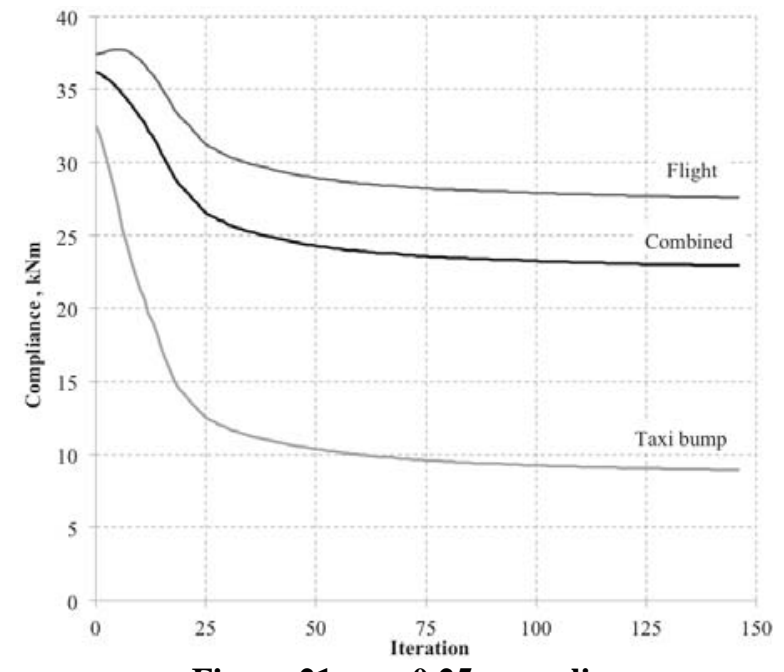

Figure 21. $\eta=0.25$, compliance.

17

American Institute of Aeronautics and Astronautics 
The solution for $\eta=0.75$ is significantly different to all previous solutions, as material is removed along the entire span of the wing, except for the tip leading edge region, where material is retained. Also, the compliance for both load cases is reduced during the optimization, Fig. 21 , in contrast to the $\eta=0.5$ result, Fig. 20. A detailed view of the solution for $\eta=0.75$ that uses cross sections to show the material distribution of the interior structure is shown in Fig. 22. The skin thickness near the trailing edge reduces in the outboard region, whereas a significant amount of material is retained in the tip leading edge region. The extra material at the tip provides inertial relief for the flight load case, although less material is added compared with the solution for pure flight loads (Fig. 8) as tip mass is penalized by the taxi-bump load case. The increase of material towards the leading edge suggests that the optimization is finding advantage from an aeroelastic effect, as the elastic axis is moved forwards. This increases the natural wash-out of the back swept wing, leading to a reduced angle of attack outboard and hence the load distribution is moved inboard. This is highlighted in Fig. 23, which shows the DLM box pressure coefficients for the steady level flight load case for the $\eta=1.0$ and $\eta=0.75$ solutions. The pressure at the tip leading edge region is lower for the $\eta=0.75$ solution, compared with the $\eta=1.0$ solution. Moving the load distribution inboard is desirable for compliance optimization and wing structure design in general, as the overall bending moment on the wing is reduced.

The weight of the $\eta=0.75$ design is the lowest of all the optimal designs obtained in this work and the compliance values for the two load cases are much closer, with the flight load compliance approximately three times the ground load compliance, Table 1. Also, the fight load compliance is only $20 \%$ greater, but the taxi-bump compliance is reduced by $77 \%$ compared with the pure flight load optimum. The results suggest that the design obtained using $\eta=0.75$ provides a good compromise between the flight and ground load cases for compliance minimization.

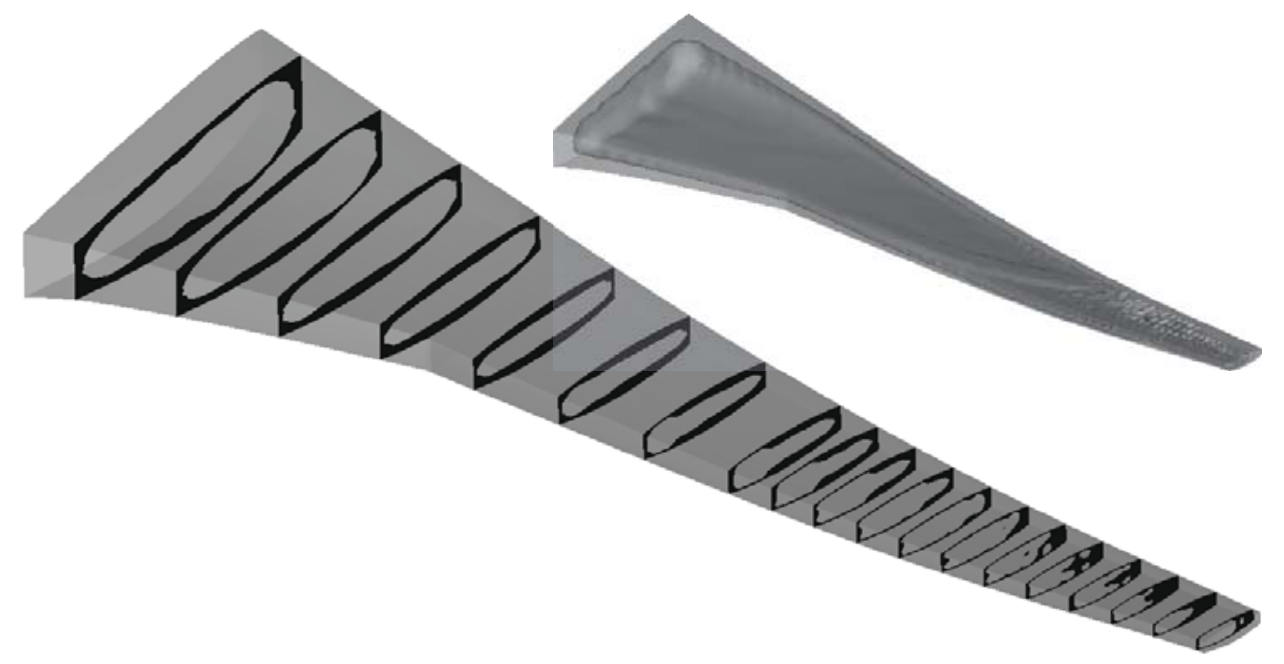

Figure 22. Cross sections through the wing box (not ribs), $\eta=0.75$ solution.

\section{Discussion}

The wing box structures obtained in this work using a 3D level set topology optimization method are very different from conventional structures. There are no spar or rib type members and the topologies mainly consist of large volumes that are either void or filled with material. There are several possible explanations for why solutions of this type are obtained with the method presented in this work. First, it appears that the solutions are often driven by the inertia effects and, for the flight load cases, the aerodynamic loads associated with the root angle of attack for trim. The wing is cantilevered from its root and, therefore, the influence of the body force loading is likely to drive the optimizer to simply add or remove material in the outboard region, where inertia effects are more significant. The angle of attack for trim is mainly influenced by the weight of the wing box, which is a global quantity and therefore unlikely to produce local features during optimization. However, the analysis and shape derivative do account for the aeroelastic feedback on the structural deformation, angle of attack and, therefore, the total compliance. The influence of the aeroelastic feedback on the optimized solutions for the examples studied in this work often appears less significant than the influence of the body force loading and total wing box weight. However, 
evidence of the aeroelastic feedback may be seen in the variations in the skin thickness for the flight load case examples and especially for the multiple load case problem with $\eta=0.75$. Further work is required to understand the influence of each factor on the optimized designs. It would also be interesting to include other loads, such as weight from fuel, engines and leading and trailing edge devices and additional aerodynamic loads from flaps and ailerons.

The role of ribs and spars in conventional wing box structures is not simply to provide stiffness and there are many constraints, such as stresses, buckling, aeroelastic stability and maintaining the aerodynamic shape of the wing. The problem studied in this work does not take into account such constraints that could drive the structure to form more spar/rib-like internal members.

A further consideration is the part of the wing box structure that is not part of the design space. The outer layer of elements was forced to remain part of the structure during the entire optimization, primarily to provide the aerodynamic surface. The mesh was reasonably coarse; therefore, the contribution of the fixed elements to the overall stiffness is significant and could be influencing the optimal design of the wing box interior. This factor could be investigated by using a finer mesh, so that the influence of the fixed outer layer of elements is reduced. Another approach could be to include the outer layer, or at least the wing skins, as part of the design space, although the skins should remain continuous, without holes, to support the aerodynamic loads.

The solutions obtained may also be influenced by the limitations of the level set based optimization method. The method employed in this work does not have a mechanism for introducing new holes. Therefore, the solutions are dependent on the initial design. ${ }^{11-13}$ This factor could be investigated by starting from different initial designs or by introducing hole creation. ${ }^{13}$

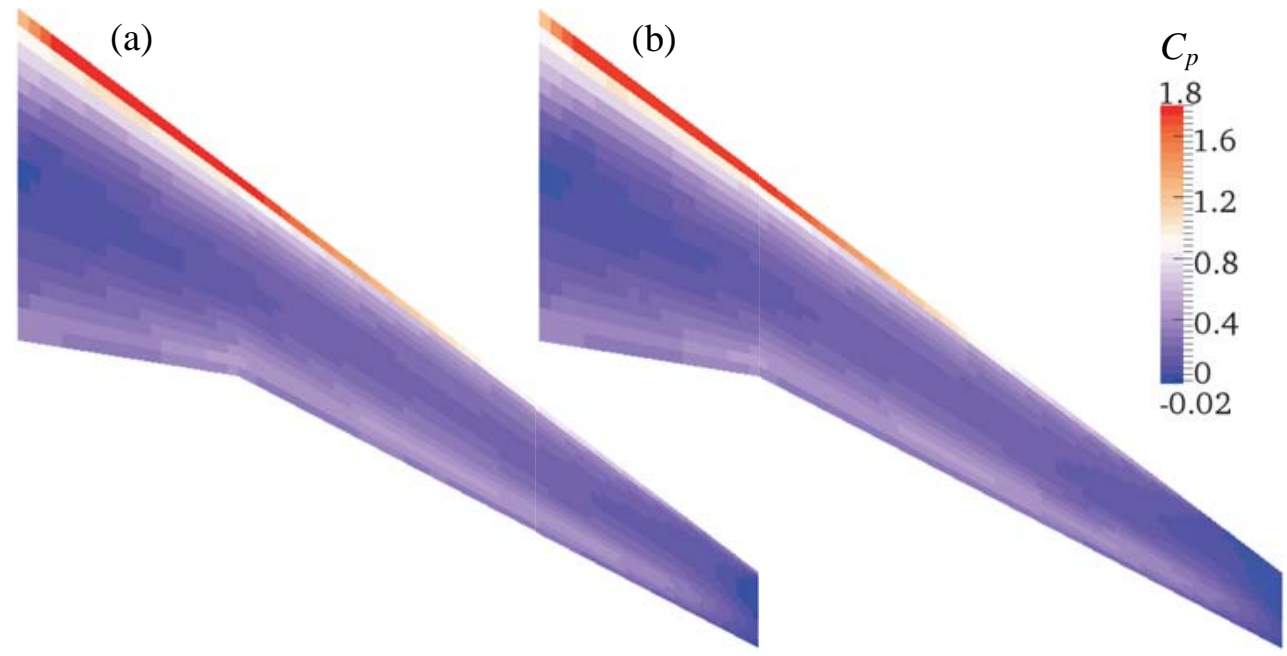

Figure 23. DLM box pressure coefficients: a) steady level flight solution, b) $\eta=0.75$ solution.

\section{Conclusions}

This paper uses level set based topology optimization to design the wing box structure of the Common Research Model. The optimizer is free to choose any structure within the 3D wing box space and the level set method is employed on a 3D unstructured grid. A fast marching method is employed and developed to efficiently compute the velocity extension and implicit function reinitialization on the unstructured grid.

The compliance of the wing box is minimized under body force and aerodynamic loading, which is coupled to the deformed wing shape. The root angle of attack is allowed to change during the optimization to meet the trim condition that weight equals lift. The adjoint method is used to obtain the shape derivative of the coupled aerostructural problem, which is then used to define the velocity function used in the level set method.

The compliance minimization problem is solved for single load cases, including steady level flight, maneuvers and a ground load tax-bump case. The solutions for the flight load cases are very similar, with material added to the outboard region for inertia relief. In contrast, the solution for the taxi-bump case removed material from the outboard region to reduce the inertial loading effect. To resolve this conflict in optimal deigns for flight and ground load cases, a multiple load case problem is formulated, where one case is steady level flight and the other is the taxibump. A load case weight factor of 0.75 (on the flight load case) provided a reasonable compromise solution for good flight and ground load performance. 
The method is able to efficiently and smoothly obtain solutions for the compliance minimization of the CRM wing box. The solutions are remarkably different from conventional rib-spar type designs, as the topologies mainly consist of large volumes that are either void or filled with material. Further refinements are suggested to investigate this, such as, the problem definition to include constraints, the modeling of the fixed part of the part of the structure and the lack of a hole creation mechanism in the level set method.

\section{Acknowledgements}

This work is funded by the Fixed Wing project under NASA's Fundamental Aeronautics Program. The authors would like to thank Dr. Maxwell Blair for his example DLM code and the Numerical Analysis Group at the Rutherford Appleton Laboratory for their FORTRAN HSL packages.

\section{References}

${ }^{1}$ Bendsøe, M. P. and Sigmund, O., Topology optimization: Theory, Methods and Applications, Springer-Verlag, Germany, 2004.

${ }^{2}$ Balabanov, V. O. and Haftka, R. T., "Topology optimization of transport wing internal structure," Journal of Aircraft, Vol. 33, No. 1, 1996, pp. 232-233.

${ }^{3}$ Maute, K., and Allen, M., "Conceptual design of aeroelastic structures by topology optimization," Structural and Multidisciplinary Optimization, Vol. 27, No. 1, 2004, pp. 27-42.

${ }^{4}$ Eschenauer, H. A. and Olhoff, N., "Topology optimization of continuum structures: a review," Applied Mechanics Reviews, Vol. 54, No. 4, 2001, pp. 331-390.

${ }^{5}$ Krog, L., Tucker, A., Kemp, M. and Boyd R., "Topology optimization of aircraft wing box ribs," 10th AIAA/ISSMO Multidisciplinary Analysis and Optimization Conference, AIAA 2004-4481, Albany, New York, 2004.

${ }^{6}$ Gomes A. A. and Suleman, A., "Topology optimization of a reinforced wing box for enhanced roll maneuvers, AIAA Journal, Vol. 46, No. 3, 2008, pp. 548-556.

${ }^{7}$ Stanford B. and Beran, P., "Optimal structural topology of a platelike wing for subsonic aeroelastic stability," Journal of Aircraft, Vol. 48, No.4, 2011, pp. 1193-1203.

${ }^{8}$ De Leon, D. M., de Souza, C. E., Fonseca, J. S. O. and da Silva, R. G. A., "Aeroelastic tailoring using fiber orientation and topology optimization," Structural and Multidisciplinary Optimization, Vol. 46, No. 5, 2012, pp. 663-677.

${ }^{9}$ Stanford B., and Ifju, P., "Aeroelastic topology optimization of membrane structures for micro air vehicles," Structural and Multidisciplinary Optimization, Vol. 38, No. 3, 2009, pp. 301-316.

${ }^{10}$ James, K. A., Aerostructural shape and topology optimization of aircraft wings, PhD Thesis, University of Toronto, Canada, 2012.

${ }^{11}$ Allaire, G., Jouve F. and Toader, A., "Structural optimization using sensitivity analysis and a level-set method," Journal of Computational Physics, Vol. 194, No. 1, 2004, pp. 363-393.

${ }^{12}$ Wang, M. Y., Wang X. and Guo, D., "A level set method for structural topology optimization," Computer Methods In Applied Mechanics and Engineering, Vol. 192, No. 1-2, 2003, pp.227-246.

${ }^{13}$ Dunning P. D. and Kim, H. A., "A new hole insertion method for level set based structural topology optimization," International Journal for Numerical Methods in Engineering, Vol. 93, No. 1, 2013, pp. 118-134.

${ }^{14}$ Deaton, J. D. and Grandhi, R. V., "A survey of structural and multidisciplinary continuum topology optimization: post 2000," Structural and Multidisciplinary Optimization, In Press. (DOI: 10.1007/s00158-013-0956-z)

${ }^{15}$ Gain, A. L. and Paulino, G. H., "A critical comparative assessment of differential equation-driven methods for structural topology optimization," Structural and Multidisciplinary Optimization, Vol. 48, No. 4, 2013, pp. 685-710.

${ }^{16}$ van Dijk, N. P., Maute, K., Langelaar, M. and van Keulen, F., "Level-set methods for structural topology optimization: a review," Structural and Multidisciplinary Optimization, Vol. 48, No. 3, 2013, pp 437-472.

${ }^{17}$ Sethian, J. A., Level set methods and fast marching methods, $2^{\text {nd }}$ ed., Cambridge University Press, New York, 1999.

${ }^{18}$ Osher, S. and Fedkiw, R., Level set methods and dynamic implicit surfaces, Springer, New York, 2003.

${ }^{19}$ Luo, J., Luo, Z., Chen, L., Tong, L., Wang, M. Y., "A semi-implicit level set method for structural shape and topology optimization,” Journal of Computational Physics, Vol. 227, No. 11, 2008, pp.5561-5581.

${ }^{20}$ Xia, Q., Wang, M. Y., Wang, S. and Chen, S., "Semi-Lagrange method for level-set-based structural topology and shape optimization," Structural and Multidisciplinary Optimization, Vol. 31, No. 6, 2006, pp. 419-429.

${ }^{21}$ Wang, S. Y. and Wang, M. Y., "Radial basis functions and level set method for structural topology optimization," International Journal For Numerical Methods In Engineering, Vol. 65, No. 12, 2006, pp. 2060-2090.

${ }^{22}$ Luo, Z., Tong, L., Wang, M. Y. and Wang, S., "Shape and topology optimization of compliant mechanisms using a parameterization level set method," Journal of Computational Physics, Vol. 227, No. 1, 2007, pp. 680-705.

${ }^{23}$ Gomes, A. A. and Suleman, A., "Application of spectral level set methodology in topology optimization," Structural and Multidisciplinary Optimization, Vol. 31, No. 6, 2006, pp. 430-443.

${ }^{24}$ Takezawa, A., Nishiwaki, S. and Kitamura, M., "Shape and topology optimization based on the phase field method and sensitivity analysis," Journal of Computational Physics, Vol. 229, No. 7, 2010, pp. 2697-2718.

${ }^{25}$ Blank L., Garcke, H., Sarbu, L., Srisupattarawanit, T., Styles, V. and Voigt, A., "Phase-field approaches to structural topology optimization," International Series of Numerical Mathematics, Vol. 160, 2010, pp. 245-256. 
${ }^{26}$ James K. A. and Martins, J. R. R. A., “An isoparametric approach to level set topology optimization using a body-fitted finite element mesh,” Computers and Structures, Vol. 90-91, 2012, pp. 97-106.

${ }^{27}$ Dunning, P. D., Brampton, C. J. and Kim, H. A., "Multidisciplinary level set topology optimization of the internal structure of an aircraft wing," 10th World Congress on Structural and Multidisciplinary Optimization, Orlando, Florida, USA, May 19-24, 2013.

${ }^{28}$ Vassberg, J., DeHaan, M., Rivers, S. and Wahls, R., "Development of a common research model for applied CFD validation studies," AIAA Applied Aerodynamics Conference, Honolulu, Hawaii, August 10-13, 2008.

${ }^{29}$ Brampton, C. J., Kim H. A. and Cunningham, J. L., "Level set topology optimisation of aircraft wing considering aerostructural interaction," 14th AIAA/ISSMO Multidisciplinary Analysis and Optimization, Indianapolis, IN USA, Sep, 2012.

${ }^{30}$ Cook, R., Malkus, D., Plesha, M. and Witt, R., Concepts and Applications of Finite Element Analysis, $4^{\text {th }}$ ed., John Wiley \& Sons, New York, 2002.

${ }^{31}$ Min, C. and Gibou, F., "Geometric integration over irregular domains with application to level-set methods," Journal of Computational Physics, Vol. 226, No. 2, 2007, pp. 1432-1443.

${ }^{32}$ Grandy, J. "Efficient computation of volume of hexahedral cells," Lawrence Livermore National Laboratory, UCRL-ID128886, Oct. 1997, doi:10.2172/632793.

${ }^{33}$ Jang, G.-W., Kim, Y. Y., and Choi, K. K., "Remesh-free shape optimization using the wavelet-Galerkin method," International Journal of Solids and Structures, Vol. 41, No.22-23, 2004, pp. 6465-6483.

${ }^{34}$ Wang, S. and Wang, M. Y., "A moving superimposed finite element method for structural topology optimization," International Journal For Numerical Methods In Engineering, Vol. 65, No. 11, 2006, pp. 1892-1922.

${ }^{35}$ Dunning, P. D., Kim H. A. and Mullineux, G., "Investigation and improvement of sensitivity computation using the areafraction weighted fixed grid FEM and structural optimization," Finite Elements in Analysis and Design, Vol. 47, No. 8, 2011, pp. 933-941.

${ }^{36}$ Sethian, J. A. and Vladimirsky, A., "Fast methods for the Eikonal and related Hamilton--Jacobi equations on unstructured meshes," Proceedings of the National Academy of Sciences of the United States of America, Vol. 97, No. 11, 2000, $5699-5703$.

${ }^{37}$ Blair, M., "A compilation of the mathematics leading to the doublet lattice method," Report No. WL-TR-92-3028, WrightPatterson Air Force Base, Ohio, 1992.

${ }^{38}$ Rodden, W. P., Taylor P. F. and McIntosh Jr., S. C, "Further refinement of the subsonic doublet-lattice method," Journal of Aircraft, Vol. 35, No. 5, 1998, pp. 720-727.

${ }^{39}$ Appa, K., "Finite-Surface Spline,” Journal of Aircraft, Vol. 26 No. 5, pp. 495-496, 1989.

${ }^{40}$ Wakayama, S. and Kroo, I., "Subsonic wing planform design using multidisciplinary optimization," Journal of Aircraft, Vol. 32, No. 4, 1995, pp. 746-753.

${ }^{41}$ Niu, M., Airframe Structural Design, Conmilit Press Ltd., Hong Kong, 1988. 